\title{
The scaling limit of the critical one-dimensional random Schrödinger operator
}

\author{
Eugene Kritchevski Benedek Valkó Bálint Virág
}

August 9, 2021

\begin{abstract}
We consider two models of one-dimensional discrete random Schrödinger operators

$$
\left(H_{n} \psi\right)_{\ell}=\psi_{\ell-1}+\psi_{\ell+1}+v_{\ell} \psi_{\ell}
$$

$\psi_{0}=\psi_{n+1}=0$ in the cases $v_{k}=\sigma \omega_{k} / \sqrt{n}$ and $v_{k}=\sigma \omega_{k} / \sqrt{k}$. Here $\omega_{k}$ are independent random variables with mean 0 and variance 1 .

We show that the eigenvectors are delocalized and the transfer matrix evolution has a scaling limit given by a stochastic differential equation. In both cases, eigenvalues near a fixed bulk energy $E$ have a point process limit. We give bounds on the eigenvalue repulsion, large gap probability, identify the limiting intensity and provide a central limit theorem.

In the second model, the limiting processes are the same as the point processes obtained as the bulk scaling limits of the $\beta$-ensembles of random matrix theory. In the first model, the eigenvalue repulsion is much stronger.
\end{abstract}

\section{Introduction}

We consider two models of one-dimensional discrete random Schrödinger operators given by the matrix

$$
H_{n}=\left(\begin{array}{cccccc}
v_{1} & 1 & & & & \\
1 & v_{2} & 1 & & & \\
& 1 & \ddots & \ddots & & \\
& & \ddots & \ddots & 1 & \\
& & & 1 & v_{n-1} & 1 \\
& & & & 1 & v_{n}
\end{array}\right)
$$


in the following two cases, referred to as the critical model and decaying model respectively:

$$
v_{k}=\sigma \omega_{k} / \sqrt{n}, \quad v_{k}=\sigma \omega_{k} / \sqrt{k} .
$$

Here $\omega_{k}$ are independent random variables with mean 0 , variance 1 and bounded third absolute moment.

We show that the eigenvectors are delocalized and the transfer matrix evolution has a scaling limit given by a stochastic differential equation. We show that in both cases eigenvalues near a fixed bulk energy $E$ have a point process limit.

We analyze the limiting point processes, in particular we give bounds on the eigenvalue repulsion, large gap probability, identify the limiting intensity and provide a central limit theorem.

In the decaying model, the limiting processes are the same as the point processes obtained as the bulk scaling limits of the $\beta$-ensembles of random matrix theory. In the critical model, the eigenvalue repulsion is much stronger.

\section{The critical model}

For very small values of $\sigma$, this matrix behaves like the discrete Laplacian - its eigenvalues are locally close to periodic and its eigenvectors are extended. The discrete measure constructed by the square of the coordinates of the normalized eigenvector will not be concentrated on any small set of points. For large $\sigma$, the matrix is close to diagonal, with eigenvalues dropped independently at random (Poisson statistics) and eigenvectors are localized. The goal of this paper is to examine the nature of the transition from extended to localized eigenvectors, and the corresponding eigenvalue statistics.

The matrix $H_{n}$ is a perturbation of the adjacency matrix of a 1-dimensional box. When the variance of $v_{k}$ does not depend on $n$, eigenvectors are localized (Carmona et al, 1987; Kunz and Souillard, 1980; Goldsheid et al, 1977) and the local statistics of eigenvalues are Poisson (Minami, 1996; Molchanov, 1981). For the perturbed adjacency matrix of higher-dimensional boxes, localization (Aizenman and Molchanov, 1993, Fröhlich and Spencer, 1983) and Poisson eigenvalue statistics (Minami, 1996) hold if the variance is a sufficiently large constant. In dimensions three and higher, for a small constant variance, it is widely conjectured that one gets random-matrix type statistics of eigenvalues and extended eigenfunctions, while for two dimensions the opinions vary.

Our regime, where the variance of the random variables $v_{\ell}$ are of order $n^{-1 / 2}$ captures the transition between localization an delocalization. We will use the methods developed in Valkó and Virág (2009) to analyze the asymptotic local spectral properties of $H_{n}$. 
If there is no noise (i.e. $\sigma=0)$ then the eigenvalues of the operator are given by $2 \cos (\pi k /(n+1))$ with $k=1, \ldots, n$. The asymptotic density near $E \in(-2,2)$ is given by $\frac{\rho}{2 \pi}$ with

$$
\rho=\rho(E)=1 / \sqrt{1-E^{2} / 4}
$$

which suggests that one should scale by $n$ near $E$ to get a meaningful limit. Thus we will study the spectrum $\Lambda_{n}$ of the scaled operator

$$
\rho n\left(H_{n}-E\right) .
$$

We will use the well-known transfer matrix description of the spectral problem for $H_{n}$. The one-dimensional eigenvalue equation $H_{n} \psi=\mu \psi$ is written as

$$
\left(\begin{array}{c}
\psi_{\ell+1} \\
\psi_{\ell}
\end{array}\right)=T\left(\mu-v_{\ell}\right)\left(\begin{array}{c}
\psi_{\ell} \\
\psi_{\ell-1}
\end{array}\right)=M_{\ell}\left(\begin{array}{c}
\psi_{1} \\
\psi_{0}
\end{array}\right)
$$

where

$$
T(x):=\left(\begin{array}{cc}
x & -1 \\
1 & 0
\end{array}\right) \text { and } M_{\ell}:=T\left(\mu-v_{\ell}\right) T\left(\mu-v_{\ell-1}\right) \cdots T\left(\mu-v_{1}\right) .
$$

Then $\mu$ is an eigenvalue of $H_{n}$ if and only if

$$
M_{n}\left(\begin{array}{l}
1 \\
0
\end{array}\right)=c\left(\begin{array}{l}
0 \\
1
\end{array}\right)
$$

for some $c \in \mathbb{R}$, or, equivalently $\left(M_{n}\right)_{11}=0$. In view of $(4)$ we parametrize $\mu=E+\frac{\lambda}{\rho n}$. We will use the notation $M_{\ell}^{\lambda}$ to emphasize dependence on $\lambda$, and use the similar notation for other quantities. Setting

$$
\varepsilon_{\ell}=\frac{\lambda}{\rho n}-\frac{\sigma \omega_{\ell}}{\sqrt{n}}
$$

we have

$$
M_{\ell}^{\lambda}=T\left(E+\varepsilon_{\ell}\right) T\left(E+\varepsilon_{\ell-1}\right) \cdots T\left(E+\varepsilon_{1}\right) \text { for } 0 \leq \ell \leq n .
$$

The scaling of $v_{\ell}=\sigma \omega_{\ell} / \sqrt{n}$ ensures that, with high probability, the transfer matrices $M_{\ell}^{\lambda}$ are bounded and the eigenfunctions are delocalized.

Theorem 1. Given $E \in(-2,2), R<\infty$ and $\sigma<\infty$, there exists a constant $c$ so that for all sufficiently large $n$ and all $t>0$, the following two statements hold with probability at least $1-c / t$. (1) We have

$$
\max _{0 \leq \ell \leq n,|\lambda| \leq R} \operatorname{Tr} M_{\ell}^{\lambda} M_{\ell}^{\lambda^{*}}<t .
$$


(2) For each eigenvector $\psi$ of $H_{n}$, normalized by $\sum_{\ell=1}^{n}\left|\psi_{\ell}\right|^{2}=1$ and corresponding to an eigenvalue $\mu \in\left[E-\frac{R}{\rho n}, E+\frac{R}{\rho n}\right]$, we have

$$
\frac{2}{(n+1) t^{2}}<\left|\psi_{\ell}\right|^{2}+\left|\psi_{\ell+1}\right|^{2}<\frac{2 t^{2}}{n+1}, \quad 0 \leq \ell \leq n
$$

In order to understand the interaction of the eigenvalues near $E$ and of the corresponding eigenvectors, one would ideally like to derive a limiting diffusion process for (8). The starting observation is that $M_{\ell}^{\lambda}$ cannot have a continuous limit. The obstacle is that for large $n$ each transfer matrix $T\left(E+\varepsilon_{k}\right)$ in $(8)$ is not close to $I$ but to $T(E)$. Thus we are led to consider, instead of $M_{\ell}^{\lambda}$, the matrices

$$
Q_{\ell}^{\lambda}=T^{-\ell}(E) M_{\ell}^{\lambda}, \quad 0 \leq \ell \leq n,
$$

which will evolve regularly.

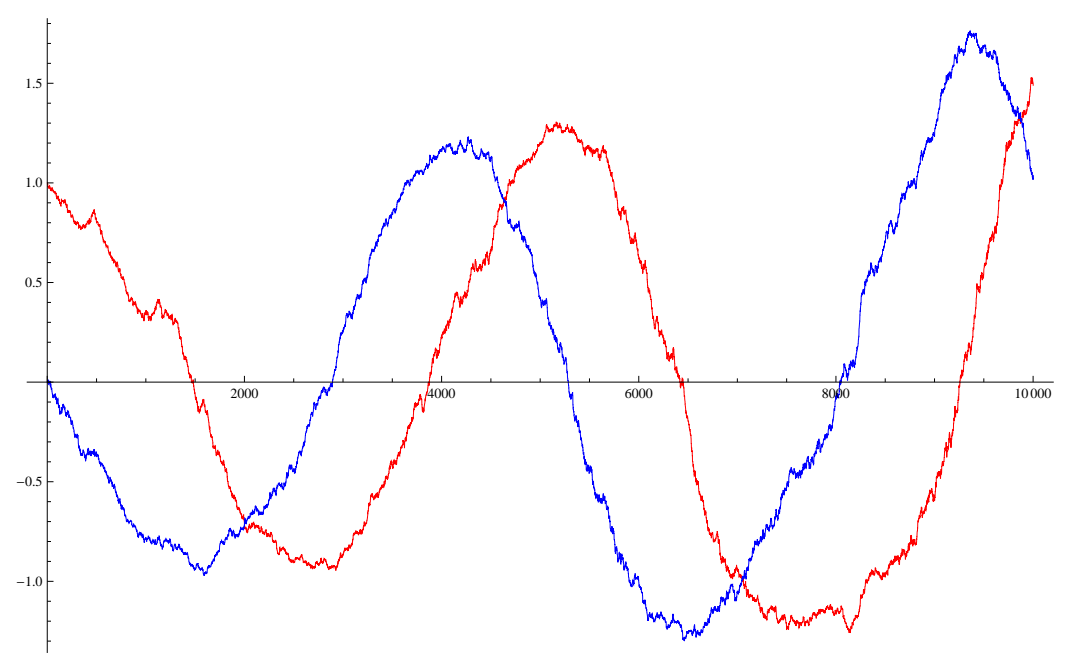

Figure 1: Simulation of the entries of the first row of $Q_{\ell}^{\lambda}$ with $E=1, \lambda=25$ and $n=10000$.

To control the correction factor $T^{-\ell}(E)$, we diagonalize $T(E)=Z D Z^{-1}$ with

$$
D=\left(\begin{array}{cc}
\bar{z} & 0 \\
0 & z
\end{array}\right), \quad Z=\left(\begin{array}{cc}
\bar{z} & z \\
1 & 1
\end{array}\right), \quad z=E / 2+i \sqrt{1-(E / 2)^{2}} .
$$

Theorem 2. Assume $0<|E|<2$. Let $\mathcal{B}(t), \mathcal{B}_{2}(t), \mathcal{B}_{3}(t)$ be independent standard Brownian motions in $\mathbb{R}, \mathcal{W}(t)=\frac{1}{\sqrt{2}}\left(\mathcal{B}_{2}(t)+i \mathcal{B}_{3}(t)\right)$. Then the stochastic differential equation

$$
d Q^{\lambda}=\frac{1}{2} Z\left(\left(\begin{array}{cc}
i \lambda & 0 \\
0 & -i \lambda
\end{array}\right) d t+\left(\begin{array}{cc}
i d \mathcal{B} & d \mathcal{W} \\
d \overline{\mathcal{W}} & -i d \mathcal{B}
\end{array}\right)\right) Z^{-1} Q^{\lambda}, \quad Q^{\lambda}(0)=I
$$


has a unique strong solution $Q^{\lambda}(t): \lambda \in \mathbb{C}, t \geq 0$, which is analytic in $\lambda$. Moreover with $\tau=(\sigma \rho)^{2}$

$$
\left(Q_{\lfloor n t / \tau\rfloor}^{\lambda}, 0 \leq t \leq \tau\right) \Rightarrow\left(Q^{\lambda / \tau}(t), 0 \leq t \leq \tau\right)
$$

in the sense of finite dimensional distributions for $\lambda$ and uniformly in $t$. Also, for any given $0 \leq t \leq \tau$ the random analytic functions $Q_{\lfloor n t / \tau\rfloor}^{\lambda}$ converge in distribution to $Q^{\lambda / \tau}(t)$ with respect to the local uniform topology.

Theorem 2 is a one-dimensional version of a more general quasi-one-dimensional theorem that appears in Valkó and Virág (2010). This proof, which predates the one in that paper, is included here for completeness. The preprint Valkó and Virág (2010) was followed by the preprint of Bachmann and De Roeck (2010), who, in independent work, also study SDE limits of transfer matrices. Their starting point the so-called DMPK theory in the physics literature, which is essentially the study of diffusive limits of quasi-one-dimensional random Schrödinger operators from a slightly different point of view. We refer the reader to Bachmann and De Roeck (2010) for a discussion of this theory. One of the novelties of our approach is that it allows for studying the dependence on the eigenvalue $\lambda$, which in turn allows us to deduce the scaling limit of the spectrum, the main focus here.

The introduction of $T^{-n}(E)$ in Theorem 2 has the effect of changing the boundary condition for each $n$, so for the next result, we have to pass to subsequences.

Corollary 3. Suppose that $n_{j}$ is a subsequence so that $z^{n_{j}}$ converges. Then $T^{n_{j}}(E) \rightarrow \tilde{T}$ and the random matrix-valued analytic functions $M_{n_{j}}^{\lambda}$ converge in distribution to $\tilde{T} Q^{\lambda / \tau}(\tau)$. Moreover, $\Lambda_{n_{j}}$ converges in law to the counting measure of the zeros of the random analytic function $\lambda \mapsto$ $\left[\tilde{T} Q^{\lambda / \tau}(\tau)\right]_{11}$.

Note that the sequence $M_{n_{j}}$ has a limit if $z^{n_{j}}$ converges. For $\Lambda_{n_{j}}$ to have a limit, only the convergence of $z^{2 n_{j}}$ is needed. If it does, then the possible limits of $T^{n_{j}}$ are $\pm \tilde{T}$ and $[\tilde{T} Q(\tau)]_{11}$ and $-[\tilde{T} Q(\tau)]_{11}$ have the same zero set.

Since $H_{n}$ is symmetric, the limiting point process will live on $\mathbb{R}$. The point process can be more effectively described by a scalar SDE. We first note that for any $a, b \in \mathbb{R}^{2}$ we have

$$
Z^{-1}\left(\begin{array}{l}
a \\
b
\end{array}\right)=\frac{\rho}{2}\left(\frac{a i-b z i}{a i-b z i}\right),
$$

so $Z^{-1}$ maps real vectors to vectors with conjugate entries. Since for $\lambda \in \mathbb{R}$ the transfer matrix $Q_{\ell}^{\lambda}$, and the limiting process $Q^{\lambda}(t)$ will also be real, we can write

$$
Z^{-1} Q^{\lambda}(t)\left(\begin{array}{l}
1 \\
0
\end{array}\right)=\left(\frac{i \psi^{\lambda}(t)}{i \psi^{\lambda}(t)}\right)
$$


for some complex numbers $\psi^{\lambda}(t)$ where $\psi^{\lambda}(0)=\rho / 2$ (the extra $i$ in the above definition makes this and some upcoming formulas nicer). We will define the phase function $\varphi^{\lambda}(t)$ by

$$
e^{i \varphi^{\lambda}(t)}=\psi^{\lambda}(t) / \overline{\psi^{\lambda}(t)}, \quad \varphi^{\lambda}(0)=0
$$

This uniquely determines $\varphi^{\lambda}(t)$ assuming that it is continuous in $t$ (as long as $\operatorname{det} Q^{\lambda}(t) \neq 0$, which follows from (13)). Itô's formula then gives an SDE for the evolution of $\varphi^{\lambda}(t)$ and we can identify the zeros of $[\tilde{T} Q(\tau)]_{11}$. This leads to another description of the point process limit of $\Lambda_{n_{j}}$.

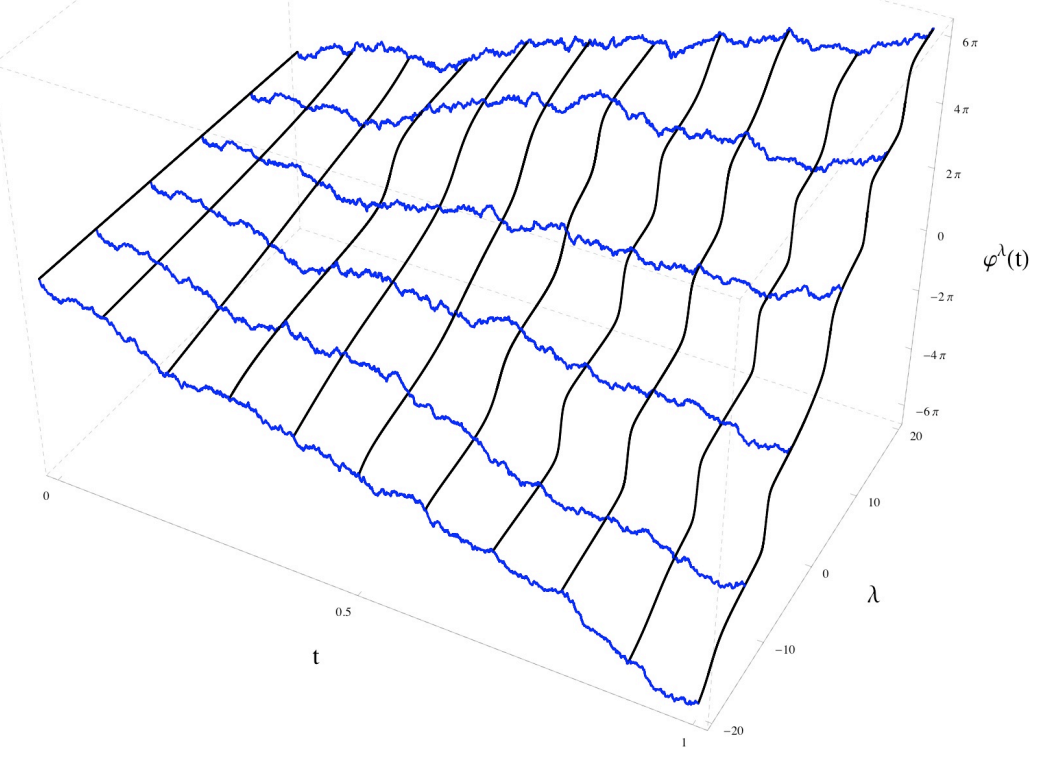

Figure 2: The phase function $\varphi^{\lambda}(t)$ for $(t, \lambda) \in[0,1] \times[-20,20]$.

Corollary 4 (Schrödinger random analytic functions). Consider the family of SDE's

$$
d \varphi^{\lambda}(t)=\lambda d t+d \mathcal{B}+\operatorname{Re}\left[e^{-i \varphi^{\lambda}(t)} d \mathcal{W}\right], \quad \varphi^{\lambda}(0)=0
$$

coupled together for all values of $\lambda \in \mathbb{R}$ where $\mathcal{B}$ and $\mathcal{W}$ are standard real and complex Brownian motions. This has a unique strong solution and for each time $t$ the function $\lambda \mapsto \varphi^{\lambda}(t)$ is strictly increasing and real-analytic with probability one.

Moreover, for $0<|E|<2$ and with $\tau=(\sigma \rho)^{2}$ the point process $\Lambda_{n}-\arg \left(z^{2 n+2}\right)-\pi$ converges in distribution to the point process

$$
\operatorname{Sch}_{\tau}:=\left\{\lambda: \varphi^{\lambda / \tau}(\tau) \in 2 \pi \mathbb{Z}\right\} .
$$


Remark 5. Note that the SDE (15) for a fixed $\lambda$ describes a Brownian motion with variance $\frac{3}{2}$ and drift $\lambda$. The random analytic function $\lambda \mapsto \varphi^{\lambda}(\tau)$ is given by the values of these coupled drifted Brownian motions at time $\tau$. In Lemma 18 we show that $\varphi^{\lambda / \tau}(\tau)+\theta \stackrel{d}{=} \varphi^{(\lambda+\theta) / \tau}(\tau)$ which means that $\operatorname{Sch}_{\tau}+\theta \stackrel{d}{=}\left\{\lambda: \varphi^{\lambda / \tau} \in \theta+2 \pi \mathbb{Z}\right\}$.

Note that the point process $\mathrm{Sch}_{\tau}$ is invariant under translation by integer multiples of $2 \pi$, but not under other translations. To fix this, we consider a translation by an independent uniform random variable:

$$
\mathrm{Sch}_{\tau}^{*}=\mathrm{Sch}_{\tau}+U[0,2 \pi]
$$

This version can be described through a variant of the the Brownian carousel introduced in Valkó and Virág (2009) (the same is true for $\mathrm{Sch}_{\tau}$, but with more complicated boundary conditions).

The Brownian carousel. Let $(\mathcal{V}(t), t \geq 0)$ be Brownian motion on the hyperbolic plane $\mathbb{H}$. Pick a point on the boundary $\partial \mathbb{H}$ and let $x^{\lambda}(0)$ equal to this point for all $\lambda \in \mathbb{R}$. Let $x^{\lambda}(t)$ be the trajectory of this point rotated continuously around $\mathcal{V}(t)$ at speed $\lambda$. Recall that Brownian motion in $\mathbb{H}$ converges to a point $\mathcal{V}(\infty)$ in the boundary $\partial \mathbb{H}$.

Theorem 6 (Brownian carousel description). We have

$$
\left\{\lambda: x^{\lambda / \tau}(\tau)=\mathcal{V}(\infty)\right\} \stackrel{d}{=} \operatorname{Sch}_{\tau}^{*}
$$

Section 3 contains the proof of Theorem 6 and a description of the ODE for $x^{\lambda}(t)$ in the Poincaré disk model of the hyperbolic plane. Amazingly, a (less complete) connection between random Schrödinger operators and Brownian motion in the hyperbolic plane had been found already in Gertsenshtein and Vasilev (1959).

Note that in the previous theorems we assumed $0<|E|<2$. The case $E=0$ is slightly different, but it gives similar results. Note that in that case $z=i$.

Theorem 7. Let $E=0$. In that case Theorem 2, and Corollaries 3 and 4 hold with the following SDE's in place of (13) and 15):

$$
d Q^{\lambda}=\frac{1}{2} Z\left(\left(\begin{array}{cc}
i \lambda & 0 \\
0 & -i \lambda
\end{array}\right) d t+\left(\begin{array}{cc}
i d \mathcal{B}_{1} & i d \mathcal{B}_{2} \\
-i d \mathcal{B}_{2} & -i d \mathcal{B}_{1}
\end{array}\right)\right) Z^{-1} Q^{\lambda}, \quad Q^{\lambda}(0)=I .
$$

and

$$
d \varphi^{\lambda}=\lambda d t+d \mathcal{B}_{1}+\cos \left(\varphi^{\lambda}\right) d \mathcal{B}_{2}+\frac{1}{4} \cos \left(2 \varphi^{\lambda}\right) d t, \quad \varphi^{\lambda}(0)=0 .
$$

Note that since $z=i$ we just need to fix the remainder of $n_{j} \bmod 4$ for $z^{n_{j}}$ to converge and the parity of $n_{j}$ for $\arg \left(z^{2 n_{j}+2}\right)$ to converge. 


\section{Properties of the limiting eigenvalue process for the critical model}

We now discuss some of the properties of the limit process $\mathbf{S c h}_{\tau}$. We describe the eigenvalue repulsion, we compute the intensity of the point process and then give the asymptotic probability of finding a large gap. We also provide a central limit theorem for the number of points in a growing interval. Let $\operatorname{Sch}_{\tau}[a, b]$ denote the number of points of $\operatorname{Sch}_{\tau}$ in the set $[a, b]$.

Theorem 8 (Eigenvalue repulsion). For $\mu \in \mathbb{R}$ and $\varepsilon>0$ we have

$$
\mathbb{P}\left\{\operatorname{Sch}_{\tau}[\mu, \mu+\varepsilon] \geq 2\right\} \leq 4 \exp \left(-(\log (\tau / \varepsilon)-\tau)^{2} / \tau\right)
$$

whenever the squared expression is nonnegative.

Remark 9. In case of the classical random matrix models GOE, GUE, GSE the eigenvalue repulsion is a lot weaker: it is of the order of $\varepsilon^{2+\beta}$ where $\beta=1,2$ and 4 in the respective cases.

Theorem 10 (Intensity of the point process). The intensity measure $A \mapsto \mathbb{E} \operatorname{Sch}_{\tau}(A)$ has density

$$
\sum_{k} p(2 \pi k+x), \quad p(y)=\frac{1}{\sqrt{3 \pi \tau}} e^{-\frac{1}{3 \tau} y^{2}}
$$

at $x$. This is the density of a centered normal random variable with variance $\frac{3}{2} \tau$ mod $2 \pi$ (a theta function).

Remark 11. If the random variables $\omega_{\ell}$ have a a bounded probability density $g(x) d x$, then the general Wegner and Minami's estimates for random discrete Schrödinger operators (Minami, 1996; Graf and Vaghi, 2007; Belissard et al, 2007; Combes et al, 2009) give

$$
\mathbb{P}\left(\Lambda_{n}\left[\mu_{1}, \mu_{2}\right] \geq 1\right) \leq \sqrt{n} \sigma^{-1}\|g\|_{\infty}\left(\mu_{2}-\mu_{1}\right)
$$

and

$$
\mathbb{P}\left(\Lambda_{n}\left[\mu_{1}, \mu_{2}\right] \geq 2\right) \leq \frac{\pi^{2}}{2} n \sigma^{-2}\|g\|_{\infty}^{2}\left(\mu_{2}-\mu_{1}\right)^{2} .
$$

Since we rescale the potential by $\sqrt{n}$, both $(20)$ and $(21)$ diverge as $n \rightarrow \infty$, whereas Theorems 8 and 10 give effective bounds. Moreover, this theorem applies to singular potentials, such as Bernoulli random variables \pm 1 with probability $1 / 2$.

Theorem 12 (Probability of large gaps). The probability that $\mathrm{Sch}_{\tau}$ has a large gap is

$$
\mathbb{P}\left(\operatorname{Sch}_{\tau}[0, \lambda]=0\right)=\exp \left\{-\frac{\lambda^{2}}{4 \tau}(1+o(1))\right\}
$$

where $o(1) \rightarrow 0$ for a fixed $\tau$ as $\lambda \rightarrow \infty$. 
The following theorem shows that for large $\lambda$ the number of points in $[0, \lambda]$ is close to a normal random variable with mean $\lambda /(2 \pi)$ and a fixed variance.

Theorem 13 (Central limit theorem). As $\lambda \rightarrow \infty$ we have

$$
\left(\varphi^{0}(\tau), \varphi^{\lambda}(\tau)-\lambda\right) \Rightarrow\left(\xi_{0}+\xi_{1}, \xi_{0}+\xi_{2}\right)
$$

where $\xi_{0}, \xi_{1}, \xi_{2}$ are independent mean zero normal random variables with variances $\tau, \tau / 2, \tau / 2$, respectively.

In particular, for $\theta \in[0,2 \pi)$ and $k \rightarrow \infty$ along the integers we have

$$
\mathrm{Sch}_{\tau}[0,2 \pi k+\theta]-k \Rightarrow\left\lfloor\frac{\xi_{0}+\xi_{2}+\theta}{2 \pi}\right\rfloor-\left\lfloor\frac{\xi_{0}+\xi_{1}}{2 \pi}\right\rfloor .
$$

\section{The decaying model}

The decaying model (2) can be thought of as the truncation of a discrete Schrödinger operator

on the infinite half line with potential $v_{k}=\sigma \omega_{k} / \sqrt{k}$. Similar operators have been studied in the literature (see Delyon et al, 1985; Kiselev et al, 1998, and references therein for earlier works). In these works, the standard deviation of the $k$ th diagonal element is on the order of $k^{-\alpha}$ for $\alpha>0$. Depending on $\alpha$, the operator has different generic spectral properties.

- Slow decay: for $0<\alpha<1 / 2$, the spectrum is pure point with probability one.

- Fast decay: for $\alpha>1 / 2$, the spectrum is absolutely continuous with probability one.

- Critical decay: for $\alpha=1 / 2$, and small enough $\sigma$, the spectrum is singular continuous on an interval $(-c, c)$ and pure point on $(-2,-c) \cup(c, 2)$, with probability one.

It is natural to investigate the fine eigenvalue statistics in these three cases. Motivated by this question, Killip and Stoiciu (2009) described the local behaviour of the spectrum in the context of random CMV matrices, the unitary analog of one-dimensional discrete Schrödinger operators.

Our decaying model corresponds to the critical case. It will be convenient to reverse the indices to have $v_{k}=\sigma \omega_{k} / \sqrt{n+1-k}$. We scale near $E \in(-2,2) \backslash\{0\}$ and define $Q_{\ell}^{\lambda}$ as before. This process will converge to an SDE similar to (13), but the convergence will only hold on $[0,1)$.

Theorem 14. We have the following limit on $[0,1)$ :

$$
d Q^{\lambda}=\frac{1}{2} Z\left(\left(\begin{array}{cc}
i \lambda & 0 \\
0 & -i \lambda
\end{array}\right) d t+\frac{\sigma \rho}{\sqrt{1-t}}\left(\begin{array}{cc}
i d \mathcal{B} & d \mathcal{W} \\
d \overline{\mathcal{W}} & -i d \mathcal{B}
\end{array}\right)\right) Z^{-1} Q^{\lambda}, \quad Q^{\lambda}(0)=I
$$

in the sense of finite dimensional distributions for $\lambda$ and uniformly on compacts in $t$. 
From (23) and Itô's formula it follows that the phase function $\varphi^{\lambda}$ (which can be defined the same way as in (14)) will satisfy the following SDE on $[0,1)$ :

$$
d \varphi^{\lambda}(t)=\lambda d t+\frac{\sigma \rho}{\sqrt{1-t}}\left(d \mathcal{B}+\operatorname{Re}\left[e^{-i \varphi^{\lambda}(t)} d \mathcal{W}\right]\right), \quad \varphi^{\lambda}(0)=0
$$

Note that since $\int_{0}^{1} \frac{1}{1-t} d t=\infty$, the process $\varphi^{\lambda}(t)$ does not have a limit as $t \rightarrow 1$. However the relative phase function $\alpha^{\lambda}=\varphi^{\lambda}-\varphi^{0}$ will converge and its limit will describe the point process limit of the spectrum.

Theorem 15. Let $\alpha^{\lambda}(t), 0 \leq t \leq 1, \alpha^{\lambda}(0)=0$, be the solution to

$$
d \alpha^{\lambda}(t)=\lambda d t+\frac{\sigma \rho}{\sqrt{1-t}}\left(\operatorname{Re}\left[\left(e^{-i \alpha^{\lambda}(t)}-1\right) d \mathcal{W}\right]\right) .
$$

The function $g(\lambda)=\frac{1}{2 \pi} \lim _{t \rightarrow 1^{-}} \alpha^{\lambda}(t)$ is integer valued and non-decreasing. If $n \rightarrow \infty$ then the scaled eigenvalue process (see (4)) converges to a point process $\Lambda$ with counting function $g$.

Applying the time change $t=1-e^{-\beta \tau / 4}$ for the SDE 25$)$ we get

$$
d \alpha^{\lambda}=\lambda \frac{\beta}{4} e^{-\frac{\beta}{4} t} d t+\operatorname{Re}\left[\left(e^{-i \alpha^{\lambda}}-1\right)\left(d \mathcal{B}_{1}+i d \mathcal{B}_{2}\right)\right], \quad \alpha^{\lambda}(0)=0, \quad t \in[0, \infty),
$$

where $\mathcal{B}_{1}, \mathcal{B}_{2}$ are independent standard Brownian motions and $\beta=\frac{2}{(\sigma \rho)^{2}}$; this is precisely the SDE that describes the Sine $_{\beta}$ process given in Valkó and Virág (2009).

Corollary 16. The point process $\Lambda$ agrees with the point process Sine $_{\beta}$, the bulk limit of the beta Hermite ensembles of random matrix theory with $\beta=\frac{2}{(\sigma \rho)^{2}}$.

The Hermite $\beta$-ensemble is a finite ensemble with joint density

$$
Z_{\beta, N}^{-1} \prod_{i<j}\left|\lambda_{i}-\lambda_{j}\right|^{\beta} e^{-\frac{\beta}{4} \sum_{i} \lambda_{i}^{2}}
$$

this suggests that the eigenvalue repulsion is of the order of $\varepsilon^{2+\beta}$ (in the sense of Theorem 8), and this can be proved using (25). In Valkó and Virág (2009) it was proved that Sine $\beta$ is translation invariant with density $(2 \pi)^{-1}$ this provides the analogue of Theorem 10 . The asymptotic probability of large gaps was identified in Valkó and Virág (2010). As $\lambda \rightarrow \infty$ we have

$$
\mathbb{P}\left(\text { Sine }_{\beta}[0, \lambda]=0\right)=\left(\chi_{\beta}+o(1)\right) \lambda^{\gamma_{\beta}} \exp \left(-\frac{\beta}{64} \lambda^{2}+\left(\frac{\beta}{8}-\frac{1}{4}\right) \lambda\right)
$$

with $\gamma_{\beta}=\frac{1}{4}\left(\frac{\beta}{2}+\frac{2}{\beta}-3\right)$ and $0<\chi_{\beta}<\infty$.

We will also prove the analogue of Theorem 13 . 
Theorem 17. As $\lambda \rightarrow \infty$ we have

$$
\frac{1}{\sqrt{\log \lambda}}\left(\operatorname{Sine}_{\beta}[0, \lambda]-\frac{\lambda}{2 \pi}\right) \Rightarrow \mathcal{N}\left(0, \frac{2}{\beta \pi^{2}}\right) .
$$

An $n \rightarrow \infty$ version of this theorem for finite matrices from circular and Jacobi $\beta$ ensembles was shown by Killip (2008).

Section 2 contains the proofs about the various properties of the limiting point processes. Section 3 will describe some connections to the Brownian carousel introduced in Valkó and Virág (2009) and prove the theorem about the carousel representation of the limiting point process. In Sections 4 and 5 we will provide the proofs for the scaling limit of the spectrum for the first model (with the constant variance) together with the delocalization of eigenvectors. Section 6 will deal with the proof in the case of the second model (with the decaying potential). Finally, the Appendix (Section 7) contains the proof for the existence of unique analytic solutions for the discussed SDEs and a technical proposition about the convergence of discrete time Markov chains to stochastic differential equations.

\section{Analysis of the limiting point process}

In this section we will provide the proofs for the theorems related to various properties of our limiting point processes.

We will first show a translation invariance property for the phase function $\varphi$.

Lemma 18 (Invariance). For every $\theta \in \mathbb{R}$ we have

$$
\varphi^{\lambda-\theta}(t)+\theta t \stackrel{d}{=} \varphi^{\lambda}(t)
$$

as functions of $\lambda, t$.

Proof. From 15 it is clear that $\tilde{\varphi}^{\lambda}(t)=\varphi^{\lambda-\theta}(t)+\theta t$ satisfies the following one parameter family of SDEs:

$$
d \tilde{\varphi}^{\lambda}(t)=\lambda d t+d \mathcal{B}+\operatorname{Re}\left[e^{-i \varphi^{\lambda-\theta}(t)} d \mathcal{W}\right], \quad \tilde{\varphi}^{\lambda}(0)=0 .
$$

Since $\varphi^{\lambda-\theta}(t)=\tilde{\varphi}^{\lambda}(t)-\theta t$ we have

$$
\operatorname{Re}\left[e^{-i \varphi^{\lambda-\theta}(t)} d \mathcal{W}\right]=\operatorname{Re}\left[e^{-i \tilde{\varphi}^{\lambda}(t)} d \widetilde{\mathcal{W}}\right], \quad \text { where } \widetilde{\mathcal{W}}(t)=\int_{0}^{t} e^{i \theta s} d \mathcal{W}
$$

is also a standard complex Brownian motion. Thus $\tilde{\varphi}^{\lambda}$ satisfies the same SDE system as $\varphi^{\lambda}$ with a different driving Brownian motion. The uniqueness of solutions shown in the Appendix implies that they indeed have the same distribution. 
In order to study the point process $\mathrm{Sch}_{\tau}$ we will use Corollary 4 . Note that for $\lambda \in \mathbb{R}$ the function $\alpha^{\lambda}(t)=\varphi^{\lambda}(t)-\varphi^{0}(t)$ (which we will call relative phase function) satisfies the following SDE system

$$
d \alpha^{\lambda}(t)=\lambda d t+\operatorname{Re}\left[\left(e^{-i \alpha^{\lambda}(t)}-1\right) d \mathcal{Z}\right], \quad \alpha^{\lambda}(0)=0,
$$

where $\mathcal{Z}$ is a standard complex Brownian motion with $d \mathcal{Z}=e^{-i \varphi^{0}} d \mathcal{W}$. For any fixed $\lambda \in \mathbb{R}$ this can be rewritten as

$$
d \alpha^{\lambda}=\lambda d t+\sqrt{2} \sin \left(\alpha^{\lambda} / 2\right) d \mathcal{B}^{\lambda}, \quad \alpha^{\lambda}(0)=0,
$$

where $\mathcal{B}^{\lambda}$ is a standard Brownian motion with $d \mathcal{B}^{\lambda}=-\sqrt{2} \operatorname{Re}\left[e^{-i \alpha^{\lambda} / 2} d \mathcal{Z}\right]$.

The quantity $\frac{1}{2 \pi} \alpha^{\lambda}(\tau)$ gives a good approximation for the number of points in $[0, \lambda]$. Indeed, by Corollary 4 we have

$$
\left|\frac{1}{2 \pi} \alpha^{\lambda / \tau}(\tau)-\operatorname{Sch}_{\tau}[0, \lambda]\right| \leq 1
$$

Proof of Theorem 8 (Eigenvalue repulsion). We will give two proofs of this theorem. The first one uses the SDE representation of Corollary 4. A second proof, at the end of Section 3, uses a geometric approach via the Brownian carousel.

For $\mu=0$ by $(30)$ we have

$$
\mathbb{P}\left(\operatorname{Sch}_{\tau}[0, \varepsilon] \geq 2\right) \leq \mathbb{P}\left(\alpha^{\varepsilon / \tau}(\tau) \geq 2 \pi\right)
$$

and the same holds for other $\mu$, with $\alpha^{\lambda / \tau}$ replaced by $\varphi^{(\lambda+\mu) / \tau}-\varphi^{\lambda / \tau}$, which satisfies the same SDE. Since this SDE is the only thing we use we can restrict our attention to $\mu=0$.

Introduce the new process $Y=\log (\tan (\alpha / 4))$ which is well defined for $\alpha \in(0,2 \pi)$. By $(29)$ and Itô's formula the process $Y$ satisfies the SDE

$$
d Y=\frac{\varepsilon / \tau}{2} \cosh (Y) d t+\frac{1}{4} \tanh (Y) d t+\frac{1}{\sqrt{2}} d B
$$

with initial condition $Y(0)=-\infty$. It is clear that $\left\{\alpha^{\varepsilon}(\tau) \geq 2 \pi\right\}=\{Y$ explodes on $[0,1]\}$. Consider now the solution $\tilde{Y}$ of the SDE 32 with initial condition $\tilde{Y}(0)=0$. By monotonicity we have

$$
Y \text { explodes on }[0, \tau] \Rightarrow \tilde{Y} \text { explodes on }[0, \tau] \Rightarrow \sup _{t \in[0, \tau]}|\tilde{Y}(t)| \geq \log (\tau / \varepsilon) \text {. }
$$

For $\varepsilon / \tau \leq 1$, the inequality $|y| \leq \log (\tau / \varepsilon)$ implies

$$
|\varepsilon / \tau \cosh (y) / 2+\tanh (y) / 4| \leq 1
$$

This means that for any $s \geq 0$ we have

$$
\sup _{t \in[0, s]}|\tilde{Y}(t)| \leq \log (\tau / \varepsilon) \Rightarrow \sup _{t \in[0, s]}\left|\tilde{Y}(t)-\frac{1}{\sqrt{2}} B(t)\right| \leq s .
$$


Let $T$ be the first hitting time of $\log (\tau / \varepsilon)$ by $\tilde{Y}$. Then by the previous argument we have $\frac{1}{\sqrt{2}}|B(T)| \geq$ $\log (\tau / \varepsilon)-T$ which leads to

$$
\begin{aligned}
\mathbb{P}\left(\sup _{t \in[0, \tau]}|\tilde{Y}(t)| \geq \log (\tau / \varepsilon)\right) & \leq \mathbb{P}\left(\frac{1}{\sqrt{2}} \sup _{t \in[0, \tau]}|B(t)| \geq \log (\tau / \varepsilon)-\tau\right) \\
& \leq 4 \exp \left(-(\log (\tau / \varepsilon)-\tau)^{2} / \tau\right) .
\end{aligned}
$$

Here the last inequality follows from Brownian scaling and

$$
\mathbb{P}\left(\sup _{t \in[0,1]}|B(t)| \geq x\right) \leq 2 \mathbb{P}\left(\sup _{t \in[0,1]} B(t) \geq x\right)=4 \mathbb{P}(B(1) \geq x) \leq 4 e^{-x^{2} / 2} .
$$

For the proof of Theorem 10 we need the following estimate.

Lemma 19. We have

$$
\mathbb{E}\left(\partial_{\lambda}^{2} \varphi^{\lambda}(t)\right)^{2}=f(t)<\infty .
$$

Proof. Differentiating (15) twice in $\lambda$ is justified by Theorem 24 in the Appendix. We get that for a fixed $\lambda$, with primes denoting $\lambda$-derivatives

$$
d \varphi^{\prime}=d t+\varphi^{\prime} d \mathcal{B}_{1}, \quad d \varphi^{\prime \prime}=\varphi^{\prime \prime} d \mathcal{B}_{1}-\varphi^{\prime 2} d \mathcal{B}_{2}, \quad \varphi^{\prime}(0)=\varphi^{\prime \prime}(0)=0
$$

where $\mathcal{B}_{1}, \mathcal{B}_{2}$ are independent real Brownian motions with variance $1 / 2$. This shows that the distribution of the first and second derivatives does not depend on $\lambda$, as we already know from the invariance Lemma 18 ,

From the first SDE we get $\mathbb{E} \varphi^{\prime}(t)=t$. Applying Itô's lemma for $\varphi^{\prime 2}$ and $\varphi^{\prime 4}$ and then using Gronwall's inequality gives that $\mathbb{E} \varphi^{\prime}(t)^{2}$ and $\mathbb{E} \varphi^{\prime}(t)^{4}$ are bounded as functions of $t$ only. Itô's lemma applied to $\varphi^{\prime \prime 2}$ gives

$$
\mathbb{E} \varphi^{\prime \prime}(t)^{2}=\int_{0}^{t} \mathbb{E}\left(\varphi^{\prime \prime}(s)^{2}+\varphi^{\prime}(s)^{4}\right) d s .
$$

Gronwall's inequality and the fact that $\mathbb{E} \varphi^{\prime 4}(s)$ is bounded leads to the desired bound.

Proof of Theorem 10 (Intensity of the point process). We will evaluate

$$
\left.\tau^{-1} \lim _{\varepsilon \rightarrow 0} \varepsilon^{-1} \mathbb{E} \#\left\{\left[\varphi^{\mu}(\tau), \varphi^{\mu+\varepsilon}(\tau)\right)\right] \cap(2 \pi \mathbb{Z})\right\}=\lim _{\varepsilon \rightarrow 0}(\tau \varepsilon)^{-1} \mathbb{E}\left[\operatorname{Sch}_{\tau}[\tau \mu, \tau(\mu+\varepsilon)]\right]
$$

By Corollary 4 the function $\varphi^{\lambda}(t)$ is analytic in $\lambda$. We will use the notation $\varpi^{\lambda}(t)=\partial_{\lambda} \varphi^{\lambda}(t)$ for the derivative. We will first evaluate the limit in (34) by switching the interval on the right with $\left[\varphi^{\mu}(\tau), \varphi^{\mu}(\tau)+\varepsilon \varpi^{\mu}(\tau)\right]$. Then we will show that the error we make is asymptotically small.

From 15 we get that $\varpi^{\lambda}$ satisfies the following SDE:

$$
d \varpi^{\lambda}=d t+\operatorname{Re}\left[\left(-i \varpi^{\lambda}\right) d \mathcal{Z}^{\lambda}\right]=d t+\varpi^{\lambda} \operatorname{Im}\left[d \mathcal{Z}^{\lambda}\right], \quad \varpi^{\lambda}(0)=0
$$


where $d \mathcal{Z}^{\lambda}=e^{-i \varphi^{\lambda}} d \mathcal{W}$. Since the SDE in 15 has the noise term $\operatorname{Re}\left[d \mathcal{Z}^{\lambda}\right]$ and the last equation has $\operatorname{Im}\left[d \mathcal{Z}^{\lambda}\right]$, the two processes are independent (for a given fixed $\lambda$ ). From the SDEs (15) and (35) we get that $\varphi^{\mu}(\tau)$ is $\mathcal{N}\left(\mu \tau, \frac{3}{2} \tau\right)$ and $\mathbb{E} \varpi^{\mu}=\tau$. Using the independence of $\varphi^{\mu}(\tau)$ and $\varpi^{\mu}$ we get

$$
\tau^{-1} \lim _{\varepsilon \rightarrow 0} \varepsilon^{-1} \mathbb{E} \#\left\{\left[\varphi^{\mu}(\tau), \varphi^{\mu}(\tau)+\varepsilon \varpi^{\mu}(\tau)\right] \cap(2 \pi \mathbb{Z})\right\}=\sum_{k \in \mathbb{Z}} p(2 k \pi+\mu \tau)
$$

where $p(\cdot)$ is the density of $\mathcal{N}\left(0, \frac{3}{2} \tau\right)$. The only thing left is to show is that

$$
\left.\lim _{\varepsilon \rightarrow 0} \varepsilon^{-1} \mathbb{E} \#\left\{\left[\varphi^{\mu}(\tau)+\varepsilon \varpi^{\mu}(\tau), \varphi^{\mu+\varepsilon}(\tau)\right)\right] \cap(2 \pi \mathbb{Z})\right\}=0
$$

We start by noting that if $Z$ is a random variable with density $f(x)$ and $x \in \mathbb{R}, y \in \mathbb{R}_{+}$then using the notation $f_{2 \pi}(x)=\sum_{k \in \mathbb{Z}} f(x+2 \pi k)$ we have

$$
\mathbb{E} \#\{[Z+x, Z+x+y] \cap(2 \pi \mathbb{Z})\}=\int_{x}^{x+y} f_{2 \pi}(-s) d s \leq|y| \max _{s \in[0,2 \pi)} f_{2 \pi}(s) .
$$

The same upper bound holds if $y<0$.

By (15) $X=\varphi^{\mu}(\tau)+\varepsilon \varpi^{\mu}(\tau)$ can be written as $X_{0}+\mathcal{B}(\tau)$ where $\mathcal{B}$ is a standard Brownian motion independent of $\mathcal{W}$ and $X_{0}$ is measurable with respect to the $\sigma$-field generated by $\mathcal{W}$. Since the density function of $\mathcal{B}(\tau) \bmod 2 \pi$ is bounded by a $\tau$ dependent constant, we may use (37) after conditioning on $\mathcal{W}$, which gives

$$
\left.\mathbb{E} \#\left\{\left[\varphi^{\mu}(\tau)+\varepsilon \varpi^{\mu}(\tau), \varphi^{\mu+\varepsilon}(\tau)\right)\right] \cap(2 \pi \mathbb{Z})\right\} \leq c \mathbb{E}\left|\varphi^{\mu+\varepsilon}(\tau)-\left(\varphi^{\mu}(\tau)+\varepsilon \varpi^{\mu}(\tau)\right)\right|
$$

To bound the right hand side, we use the integral form of the remainder in the Taylor expansion

$$
\mathbb{E}\left|\varphi^{\mu+\varepsilon}(\tau)-\left(\varphi^{\mu}(\tau)+\varepsilon \varpi^{\mu}(\tau)\right)\right| \leq \varepsilon \int_{\mu}^{\mu+\varepsilon} \mathbb{E}\left|\partial_{\lambda}^{2} \varphi^{x}(\tau)\right| d x \leq c \varepsilon^{2}
$$

In the last step we used the Cauchy-Schwarz inequality and Lemma 19, This proves (36) and completes the proof of Theorem 10 .

Proof Theorem 12 (Probability of large gaps). Let $\alpha=\alpha^{\lambda / \tau}$. We bound the desired probability in terms of phase function events:

$$
\mathbb{P}\left(\varphi^{0}(\tau) \in(0, \varepsilon) \bmod 2 \pi \text { and } \alpha(\tau) \leq 2 \pi-\varepsilon\right) \leq \mathbb{P}\left(\operatorname{Sch}_{\tau}[0, \lambda]=0\right) \leq \mathbb{P}(\alpha(\tau) \leq 2 \pi)
$$

which is clear from Corollary 4 and the definition of $\alpha$. To get a lower bound first note that we have $\varphi^{0}(\tau)=\mathcal{B}(\tau)-\operatorname{Re}[\mathcal{Z}(\tau)]$ where $\mathcal{Z}$ is the driving Brownian motion in the SDE $[28$ for $\alpha$. Since $\mathcal{B}$ is independent of $\mathcal{Z}$ we have

$$
\mathbb{E}\left[\mathbf{1}\left\{\varphi^{0}(\tau) \in(0, \varepsilon) \bmod 2 \pi\right\} \mid \mathcal{Z}\right] \geq \varepsilon \min _{x} f_{2 \pi}(x)=\varepsilon c_{\tau}>0
$$


where $f_{2 \pi}(x)$ is the density of $\mathcal{B}(\tau) \bmod 2 \pi$. This means that the lower bound in $(38)$ can be estimated with $\varepsilon c_{\tau} \mathbb{P}(\alpha(\tau) \leq 2 \pi-\varepsilon)$ from below.

Recall the SDE 29 :

$$
d \alpha=(\lambda / \tau) d t+\sqrt{2} \sin (\alpha / 2) d \mathcal{B}, \quad \alpha(0)=0
$$

In Theorem 13 of Valkó and Virág (2009) the authors analyze $\lim _{t \rightarrow \infty} P\left(\tilde{\alpha}^{\lambda}(t) \leq 2 \pi\right)$ for a similar SDE:

$$
d \tilde{\alpha}=\lambda f d t+2 \sin (\tilde{\alpha} / 2) d B, \quad \tilde{\alpha}(0)=0
$$

and with certain weak assumptions on $f$ they get the asymptotics $\exp \left(-\lambda^{2}\|f\|_{2}^{2} / 8+o(1)\right)$. The exact same methods with $f=\frac{2}{\tau} \mathbf{1}_{[0, \tau / 2]}$ in the present case give

$$
\mathbb{P}(\alpha(\tau) \leq(2-\varepsilon) \pi) \geq \exp \left\{-\frac{\lambda^{2}}{4 \tau}(1+o(1))\right\}, \quad \mathbb{P}(\alpha(\tau) \leq 2 \pi) \leq \exp \left\{-\frac{\lambda^{2}}{4 \tau}(1+o(1))\right\}
$$

We omit the straightforward details.

The asymptotic gap probability for the $\mathrm{Sine}_{\beta}$ process (see formula (27)) was analyzed to higher precision in Valkó and Virág (2010). Those techniques may also work here, resulting in an asymptotic expansion of the gap probability. It would be interesting to see how the more precise asymptotics compare to the $\beta$-ensemble case.

Proof of Theorem 13 (Central limit theorem).

By 15 we have $\left(\varphi^{0}(\tau), \varphi^{\lambda}(\tau)-\lambda\right)=\left(\xi_{0}+\xi_{1}, \xi_{0}+\xi_{2}\right)$ where

$$
\xi_{0}=\mathcal{B}(\tau), \quad \xi_{1}=\int_{0}^{\tau} \operatorname{Re}\left[e^{-i \varphi^{0}(t)} d \mathcal{W}\right], \quad \xi_{2}=\int_{0}^{\tau} \operatorname{Re}\left[e^{-i \varphi^{\lambda}(t)} d \mathcal{W}\right] .
$$

Clearly, $\xi_{0}, \xi_{1}$ and $\xi_{2}$ are Gaussians with the appropriate means and variances and $\xi_{0}$ is independent of $\left(\xi_{1}, \xi_{2}\right)$. However, the joint distribution of $\left(\xi_{1}, \xi_{2}\right)$ is not Gaussian. So we need to prove that as $\lambda \rightarrow \infty$ the joint weak limit of $\left(\xi_{1}, \xi_{2}\right)$ exists and it is given by a pair of independent normals. Let $\mathcal{Z}(t)=\int_{0}^{t} e^{-i \varphi^{0}(s)} d \mathcal{W}$ and $\mathcal{Z}=\frac{1}{\sqrt{2}}\left(\mathcal{B}_{1}+i \mathcal{B}_{2}\right)$ then

$$
\xi_{1}=\frac{1}{\sqrt{2}} \int_{0}^{\tau} d \mathcal{B}_{1}, \quad \xi_{2}=\frac{1}{\sqrt{2}} \int_{0}^{\tau} \cos \left(\alpha^{\lambda}\right) d \mathcal{B}_{1}+\frac{1}{\sqrt{2}} \int_{0}^{\tau} \sin \left(\alpha^{\lambda}\right) d \mathcal{B}_{2}:=\xi_{2,1}+\xi_{2,2} .
$$

We will show that $\left(\xi_{1}, \xi_{2,1}, \xi_{2,2}\right)$ converges weakly to three independent mean zero normals with variances $\tau / 2, \tau / 4, \tau / 4$. It is enough to prove that for any $\left(a_{1}, a_{2}, a_{3}\right) \in \mathbb{R}^{3}$ the random variable $v^{\lambda}=a_{1} \xi_{1}+a_{2} \xi_{2,1}+a_{3} \xi_{2,2}$ converges to a mean zero normal with variance $\tau\left(a_{1}^{2} / 2+a_{2}^{2} / 4+a_{3}^{2} / 4\right)$. By representing the Brownian integral as a time changed Brownian motion we can see that $v^{\lambda}$ has the 
same distribution as $\hat{\mathcal{B}}\left(\frac{1}{2} \int_{0}^{\tau}\left(a_{1}+a_{2} \cos \left(\alpha^{\lambda}\right)\right)^{2}+a_{3}^{2} \sin \left(\alpha^{\lambda}\right)^{2} d t\right)$ for some standard Brownian motion $\hat{\mathcal{B}}$. All we need to show is that

$$
2 \int_{0}^{\tau}\left(a_{1}+a_{2} \cos \left(\alpha^{\lambda}\right)\right)^{2}+a_{3}^{2} \sin \left(\alpha^{\lambda}\right)^{2} d t \rightarrow \tau\left(2 a_{1}^{2}+a_{2}^{2}+a_{3}^{2}\right) \quad \text { in probability. }
$$

Using $\cos (x)^{2}=(\cos (2 x)+1) / 2$ and $\sin (x)^{2}=(1-\cos (2 x)) / 2$ this reduces to

$$
\int_{0}^{\tau} \cos \left(2 \alpha^{\lambda}\right) d t=\operatorname{Re} \int_{0}^{1} e^{2 i \alpha^{\lambda}} d t \rightarrow 0, \quad \int_{0}^{\tau} \cos \left(\alpha^{\lambda}\right) d t=\operatorname{Re} \int_{0}^{\tau} e^{i \alpha^{\lambda}} d t \rightarrow 0
$$

in probability. We work out the second claim, as the first one can be done the same way. Using (29) and Itô's formula we get

$$
\frac{1}{i \lambda} d\left(e^{i \alpha^{\lambda}}\right)=e^{i \alpha_{\lambda}} d t+\frac{\sqrt{2}}{\lambda} \sin \left(\alpha^{\lambda} / 2\right) d \mathcal{B}^{\lambda}+\frac{i}{\lambda} \sin \left(\alpha^{\lambda} / 2\right)^{2} d t
$$

and

$$
\int_{0}^{\tau} e^{i \alpha^{\lambda}} d t=\frac{1}{i \lambda}\left(e^{i \alpha_{\lambda}(\tau)}-1\right)-\frac{\sqrt{2}}{\lambda} \int_{0}^{\tau} \sin \left(\alpha^{\lambda} / 2\right) d \mathcal{B}^{\lambda}+\frac{i}{\lambda} \int_{0}^{\tau} \sin \left(\alpha^{\lambda} / 2\right)^{2} d t
$$

As $\lambda \rightarrow \infty$ the first and third terms converge to 0 a.s., while the second term converges to 0 in $L^{2}$. This means that their sum will converge to 0 in probability which is what we needed to prove the joint limit theorem for $\left(\varphi^{0}(\tau), \varphi^{\lambda}(\tau)-\lambda\right)$.

The limit 22 follows from

$$
\operatorname{Sch}_{\tau}[0,2 \pi k+\theta]-k=\#\left\{\left[\varphi^{0}(\tau), \varphi^{\lambda / \tau}(\tau)\right] \cap 2 \pi \mathbb{Z}\right\}-k=\left\lfloor\frac{\varphi^{\lambda / \tau}(\tau)-(2 k \pi+\theta)+\theta}{2 \pi}\right\rfloor-\left\lfloor\frac{\varphi^{0}(\tau)}{2 \pi}\right\rfloor,
$$

where we also used that $\varphi^{\lambda}(t)$ is increasing in $\lambda$.

The proof of Theorem 17 is very similar.

Proof 17 (Central limit theorem for $\mathrm{Sine}_{\beta}$ ). We will consider (26) recalling that $\mathcal{Z}$ is a complex Brownian motion with independent standard real and imaginary parts and hence

$$
d \alpha^{\lambda}=\lambda \frac{\beta}{4} e^{-\frac{\beta}{4} t} d t+2 \sin \left(\alpha^{\lambda} / 2\right) d \mathcal{B}, \quad \alpha^{\lambda}(0)=0 \quad t \in[0, \infty)
$$

First note that $\tilde{\alpha}(t)=\alpha^{\lambda}(T+t)$ with $T=\frac{4}{\beta} \log (\beta \lambda / 4)$ satisfies the same SDE with $\lambda=1$. Therefore

$$
\frac{\alpha^{\lambda}(\infty)-\alpha^{\lambda}(T)}{\sqrt{\log (\lambda)}} \rightarrow 0
$$

in probability. So it suffices to find the the weak limit of

$$
\frac{\alpha^{\lambda}(T)-\lambda}{2 \pi \sqrt{\log \lambda}} .
$$


We have

$$
\alpha(T)-\lambda=-\frac{4}{\beta}+\int_{0}^{T} 2 \sin \left(\alpha^{\lambda} / 2\right) d \mathcal{B}
$$

which means

$$
\alpha(T)-\lambda+\frac{4}{\beta} \stackrel{d}{=} \hat{\mathcal{B}}\left(\int_{0}^{T} 4 \sin \left(\alpha^{\lambda} / 2\right)^{2} d t\right)
$$

for a certain standard Brownian motion $\hat{\mathcal{B}}$. In order to prove the required limit in distribution we only need to show that $\frac{4}{\log \lambda} \int_{0}^{T} \sin \left(\alpha^{\lambda} / 2\right)^{2} d t \rightarrow \frac{8}{\beta}$ in probability. We have

$$
\frac{4}{\log \lambda} \int_{0}^{T} \sin \left(\alpha^{\lambda} / 2\right)^{2} d t=\frac{8 \log [\beta \lambda / 4]}{\beta \log \lambda}+\frac{2}{\beta \log \lambda} \int_{0}^{T} \cos \left(\alpha^{\lambda}\right) d t .
$$

The first term converges to $8 / \beta$. To bound the second term we compute

$$
\begin{aligned}
\frac{4}{i \beta \lambda \log \lambda} d\left(e^{i \alpha^{\lambda}+\beta t / 4}\right)= & \frac{e^{i \alpha^{\lambda}}}{\log \lambda} d t+\frac{8}{\beta \lambda \log \lambda} e^{i \alpha^{\lambda}+\beta t / 4} \sin \left(\alpha^{\lambda} / 2\right) d \mathcal{B} \\
& +\frac{8 i}{\beta \lambda \log \lambda} e^{i \alpha^{\lambda}+\beta t / 4} \sin \left(\alpha^{\lambda} / 2\right)^{2} d t+\frac{1}{i \lambda \log \lambda} e^{i \alpha^{\lambda}+\beta t / 4} d t .
\end{aligned}
$$

The integral of the left hand side is $\frac{4}{i \beta \lambda \log \lambda}\left[4 e^{i \alpha^{\lambda}(T)} \lambda / \beta-1\right]=O\left((\log \lambda)^{-1}\right)$. The integrals of the last two terms in the right hand side are of the order of $(\lambda \log \lambda)^{-1} \int_{0}^{T} e^{\beta t / 4} d t=O\left((\log \lambda)^{-1}\right)$. Finally, the integral of the second term on the right has an $L^{2}$ norm which is bounded by $C(\log \lambda)^{-1}$. This means the integral of the first term on the right, $(\log \lambda)^{-1} \int_{0}^{T} e^{i \alpha^{\lambda}} d t$ converges to 0 in probability from which the statement of the theorem follows.

\section{The Brownian carousel}

The SDE system 28) has a geometric interpretation using the Brownian carousel introduced in Valkó and Virág (2009). Recall the SDE system (28)

$$
d \alpha^{\lambda}(t)=\lambda d t+\operatorname{Re}\left[\left(e^{-i \alpha^{\lambda}(t)}-1\right) d \mathcal{Z}\right], \quad \alpha^{\lambda}(0)=0
$$

Here $\mathcal{Z}$ is a standard complex Brownian motion.

Consider the hyperbolic plane, let $x_{0}$ be a point on the boundary and let $\mathcal{V}(t), t \geq 0$ be hyperbolic Brownian motion. For a given $\lambda \in \mathbb{R}$ we rotate the boundary point $x_{0}$ about the moving center $\mathcal{V}(t)$ with a constant angular speed $\lambda$ and denote its position by $x^{\lambda}(t)$. This is the Brownian carousel with constant speed function 1 and in Section 2 of Valkó and Virág (2009) it was proved that the hyperbolic angle determined by the points $x_{0}, \mathcal{V}(t), x^{\lambda}(t)$ satisfies the SDE (40).

The evolution of $x^{\lambda}(t)$ can be described by an ODE. Consider the Poincaré disk model for the hyperbolic plane. Then the boundary points are points on the unit circle which can be described 
by an angle $x^{\lambda}(t)=e^{i \gamma^{\lambda}(t)}$. The hyperbolic Brownian motion $\mathcal{V}$ in this model satisfies the following SDE:

$$
d \mathcal{V}=\frac{1-|\mathcal{V}|^{2}}{2} d \mathcal{Y}
$$

where $\mathcal{Y}$ is a standard complex Brownian motion. If we set $x_{0}=1, \gamma^{\lambda}(0)=0$ then

$$
\partial_{t} \gamma^{\lambda}=\lambda \frac{\left|e^{i \gamma^{\lambda}}-\mathcal{V}\right|^{2}}{1-|\mathcal{V}|^{2}}, \quad \gamma^{\lambda}(0)=0 .
$$

It is clear that this ODE system has a unique solution which is analytic and strictly increasing in $\lambda$ for any $t>0$. Note that one usually cannot get $\alpha^{\lambda}(t)$ from $\gamma^{\lambda}(t)$, however $\alpha^{\lambda}(t) \in 2 \pi \mathbb{Z}$ if and only if $\gamma^{\lambda}(t) \in 2 \pi \mathbb{Z}$.

Next we will prove Theorem 6 , if we add a random shift to $\mathrm{Sch}_{\tau}$ then the resulting point process can be described with a Brownian carousel.

Proof of Theorem 6. Let $U$ be uniform on $[0,2 \pi]$ and independent of $\operatorname{Sch}_{\tau}$. By Corollary 4 and Remark 5 the point process $\mathrm{Sch}_{\tau}+U$ has the same distribution as the solutions of the equation $\varphi^{\lambda / \tau}(\tau)=U \bmod 2 \pi$. This can be rewritten as

$$
\alpha^{\lambda}(\tau)=-\varphi^{0}(\tau)+U \bmod 2 \pi
$$

Since $U$ is independent of $\alpha^{\lambda}$ and $\varphi^{0}$, we have

$$
\left(\alpha^{\lambda}(\tau),-\varphi^{0}(\tau)+U\right) \quad \bmod 2 \pi \stackrel{d}{=} \quad\left(\alpha^{\lambda}(\tau), U\right) \quad \bmod 2 \pi
$$

Thus we can just look at the solutions of $\alpha^{\lambda}(\tau)=U \bmod 2 \pi$.

For a given $u \in[0,2 \pi)$ the solution set of $\alpha^{\lambda}(\tau)=u \bmod 2 \pi$ can be described by the carousel construction: it is given by the set of those $\lambda \in \mathbb{R}$ for which the hyperbolic angle $x_{0}, \mathcal{V}(T), x^{\lambda}(T)$ is equal to $u$.

By the Markov property of hyperbolic Brownian motion, the hyperbolic angle $x_{0}, \mathcal{V}(T), \mathcal{V}(\infty)$ is just uniform and independent of $V$ on the time interval $[0, T]$, so we may as well call it $U$. The claim follows.

We also provide an alternate proof to a version of Theorem 8 using the Brownian carousel.

Theorem 20 (Eigenvalue repulsion). For $\mu \in \mathbb{R}$ and $\varepsilon>0$ we have

$$
\mathbb{P}\left\{\operatorname{Sch}_{\tau}[\mu, \mu+\varepsilon] \geq 2\right\} \leq 4 \exp \left(-\frac{(\log (2 \pi / \varepsilon)-\tau-1)^{2}}{\tau}\right) .
$$

whenever the squared expression is nonnegative. 
Proof. As in the first proof we can assume that $\mu=0$. By (31) if there are at least two points in $[0, \varepsilon]$ then the relative phase function must be at least $2 \pi$ which means that the Brownian carousel had to take at least one full turn. Thus

$$
\mathbb{P}\left\{\operatorname{Sch}_{\tau}[0, \varepsilon] \geq 2\right\} \leq \mathbb{P}\left\{\alpha^{\varepsilon / \tau}(\tau) \geq 2 \pi\right\}=\mathbb{P}\left\{\gamma^{\varepsilon / \tau}(\tau) \geq 2 \pi\right\}
$$

where $\gamma$ is the solution of 42 . From 42 we get

$$
\gamma^{\varepsilon / \tau}(\tau) \leq \max _{0 \leq t \leq \tau}\left(1-\left|\mathcal{V}_{t}\right|^{2}\right)^{-1}=\left(1-\max _{0 \leq t \leq \tau}\left|\mathcal{V}_{t}\right|^{2}\right)^{-1}
$$

which means that

$$
\gamma^{\varepsilon / \tau}(\tau) \geq 2 \pi \quad \Rightarrow \quad 1-\frac{\varepsilon}{2 \pi} \leq \max _{0 \leq t \leq \tau}\left|\mathcal{V}_{t}\right|^{2}
$$

In the Poincaré disk model the hyperbolic distance between the origin and a point $z$ in the unit disk is given by $q(z)=\log \left(\frac{1+|z|}{1-|z|}\right)$. Thus 44 implies

$$
\max _{0 \leq t \leq \tau} q\left(\mathcal{V}_{t}\right) \geq \log (2 \pi / \varepsilon)
$$

The probability that the hyperbolic Brownian motion leaves a ball with a large radius $r$ in a fixed time is comparable to the probability that a one-dimensional Brownian motion leaves $[-r, r]$ in the same time. This follows by noting that Itô's formula with 41) gives

$$
d q=\frac{d B}{\sqrt{2}}+\frac{\operatorname{coth}(q)}{4} d t
$$

for the evolution of $q(\mathcal{V})$ with a standard Brownian motion $B$. By increasing the drift from $\operatorname{coth}(q) / 4$ to $\infty \mathbf{1}_{q \in[0,1]}+\operatorname{coth}(1) / 4$ we see that $q$ is stochastically dominated by $1+t \operatorname{coth}(1) / 4+|B(t)| / \sqrt{2}$ where $B$ is standard Brownian motion and $\operatorname{coth}(1)<4$. Thus

$$
\begin{aligned}
\mathbb{P}\left(\max _{0 \leq t \leq \tau} q\left(\mathcal{V}_{t}\right) \geq \log (2 \pi / \varepsilon)\right) & \leq \mathbb{P}\left(\max _{0 \leq t \leq \tau}|B(t)| \geq \log (2 \pi / \varepsilon)-1-\tau\right) \\
& \leq 4 \exp \left(-\frac{(\log (2 \pi / \varepsilon)-\tau-1)^{2}}{\tau}\right)
\end{aligned}
$$

which proves the theorem.

\section{Convergence of the regularized transfer matrix evolution}

This section is devoted to the proof of Theorem 2. In order to keep the notation simple, we will only treat the case when $m=1$, i.e. when a single value $\lambda \in \mathbb{C}$ is fixed. The extension to $\underline{\lambda}=\left(\lambda_{1}, \cdots, \lambda_{m}\right)$ for $m>1$ is straightforward. We drop $\lambda$ from the notation. The identity

$$
T(y) T^{-1}(x)=I+\left(\begin{array}{cc}
0 & y-x \\
0 & 0
\end{array}\right)
$$


and the recursion $M_{\ell}=T\left(E+\varepsilon_{\ell}\right) M_{\ell-1}$ from (8) implies

$$
\begin{aligned}
Q_{\ell} & =T^{-\ell}(E)\left\{T\left(E+\varepsilon_{\ell}\right) T^{-1}(E)\right\} T^{\ell}(E) Q_{\ell-1} \\
& =T^{-\ell}(E)\left(\begin{array}{cc}
1 & \varepsilon_{\ell} \\
0 & 1
\end{array}\right) T^{\ell}(E) Q_{\ell-1} .
\end{aligned}
$$

This shows that $Q_{\ell}, 0 \leq \ell \leq n$ is a Markov chain, the initial term $Q_{0}=I$. As $E$ is fixed, from now on we write $T$ for $T(E)$. We will work in the basis diagonalizing $T$ i.e. we consider $X_{\ell}=Z^{-1} Q_{\ell} Z$ instead of $Q_{\ell}$. (We have learned that such a change of basis has been considered for a slightly different problem by Schulz-Baldes (2004)). Using $T=Z D Z^{-1}$ from (12), we obtain after simplification

$$
T^{-\ell}\left(\begin{array}{cc}
0 & \varepsilon_{\ell} \\
0 & 0
\end{array}\right) T^{\ell}=\frac{i \rho \varepsilon_{\ell}}{2} Z O_{\ell} Z^{-1}, \quad O_{\ell}:=\left(\begin{array}{cc}
1 & z^{2 \ell} \\
-\bar{z}^{2 \ell} & -1
\end{array}\right) .
$$

Therefore $X_{\ell}$ is a Markov chain with the initial condition $X_{0}=I$ and given by the recurrence

$$
X_{\ell}=X_{\ell-1}+U_{\ell} X_{\ell-1}, \quad U_{\ell}=U_{\ell}^{n}:=i \rho \varepsilon_{\ell} O_{\ell} / 2
$$

Because of the oscillating factors $z^{ \pm(2 \ell)}$, the term $U_{\ell} X_{\ell-1}$ is too rough to approximate a stochastic differential. However, on a mesoscopic scale $1 \ll K \ll n$, the difference $X_{\ell+K}-X_{\ell}=\sum_{j=1}^{K} U_{\ell+j} X_{\ell+j-1}$ becomes a good approximation for a stochastic differential because the oscillations cancel in the sum. For convenience of the reader, we first present a heuristic derivation of the limiting SDE and then we give a rigorous proof.

Heuristic proof. As $X_{\ell+K}=\left(I+U_{\ell+K}\right) \cdots\left(I+U_{\ell+2}\right)\left(I+U_{\ell+1}\right) X_{\ell} \simeq\left(I+\sum_{j=1}^{K} U_{\ell+j}\right) X_{\ell}$, we have

$$
X_{\ell+K}-X_{\ell} \simeq \sum_{j=1}^{K} U_{\ell+j} X_{\ell}=\frac{i \rho}{2}\left(\sum_{j=1}^{K} \varepsilon_{\ell+j} O_{\ell+j}\right) X_{\ell}
$$

We look separately at the drift and the noise contributions, i.e. we split

$$
\frac{i \rho}{2} \sum_{j=1}^{K} \varepsilon_{\ell+j} O_{\ell+j}=\left(\frac{i \lambda}{2 n} \sum_{j=1}^{K} O_{\ell+j}\right)+\left(-\frac{i \sigma \rho}{2 n^{1 / 2}} \sum_{j=1}^{K} \omega_{\ell+j} O_{\ell+j}\right):=\mathcal{D}+\mathcal{N}
$$

Since $-2<E<2$, we have $|z|=1$ and $z^{2} \neq 1$, which implies that $\sum_{j=1}^{K} z^{ \pm(2 \ell+2 j)}$ is bounded for large $K$. With $\Delta t=K / n$ we then have

$$
\mathcal{D} \simeq \frac{\lambda \Delta t}{2}\left(\begin{array}{cc}
i & 0 \\
0 & -i
\end{array}\right)
$$


For the noise term we write

$$
\mathcal{N}=\frac{-i \sigma \rho}{2 K^{1 / 2}} \sqrt{\Delta t} \sum_{j=1}^{K} \omega_{\ell+j} O_{\ell+j}=\frac{\sigma \rho}{2} \sqrt{\Delta t}\left(\begin{array}{cc}
i \xi_{\ell, K} & \zeta_{\ell, K} \\
\bar{\zeta}_{\ell, K} & -i \xi_{\ell, K}
\end{array}\right)
$$

with

$$
\xi_{\ell, K}=-K^{-1 / 2} \sum_{j=1}^{K} \omega_{\ell+j}, \quad \zeta_{\ell, K}=-i K^{-1 / 2} \sum_{j=1}^{K} \omega_{\ell+j} z^{2 \ell+2 j} .
$$

In the limit $K \rightarrow \infty,\left(\xi_{\ell, K}, \operatorname{Re} \zeta_{\ell, K}, \operatorname{Im} \zeta_{\ell, K}\right)$ is a mean zero Gaussian vector $\left(\xi_{\ell}, \operatorname{Re} \zeta_{\ell}, \operatorname{Im} \zeta_{\ell}\right)$ whose distribution is determined by the covariance matrix. Computing the covariance matrix is equivalent to computing the limits of the expectations of $\xi_{\ell, K}^{2}, \xi_{\ell, K}, \zeta_{\ell, K}, \zeta_{\ell, K}^{2}$ and $\left|\zeta_{\ell, K}\right|^{2}$ since $\mathbb{E} \operatorname{Re} \zeta \operatorname{Im} \zeta=\frac{1}{2} \operatorname{Im} \mathbb{E} \zeta^{2}$, $\mathbb{E}(\operatorname{Re} \zeta)^{2}=\frac{1}{2}\left(\mathbb{E}|\zeta|^{2}+\operatorname{Re} \mathbb{E} \zeta^{2}\right), \mathbb{E}(\operatorname{Im} \zeta)^{2}=\frac{1}{2}\left(\mathbb{E}|\zeta|^{2}-\operatorname{Re} \mathbb{E} \zeta^{2}\right), \mathbb{E} \xi \operatorname{Re} \zeta=\operatorname{Re} \mathbb{E} \xi \zeta$ and $\mathbb{E} \xi \operatorname{Im} \zeta=\operatorname{Im} \mathbb{E} \xi \zeta$ Using 47 we get

$$
\mathbb{E} \xi_{\ell}^{2}=\mathbb{E}\left|\zeta_{\ell}^{2}\right|=1, \quad \mathbb{E} \xi_{\ell} \zeta_{\ell}=\lim _{K \rightarrow \infty} i K^{-1} \sum_{j=1}^{K} z^{2 \ell+2 j} \quad \text { and } \quad \mathbb{E} \zeta_{\ell}^{2}=-\lim _{K \rightarrow \infty} K^{-1} \sum_{j=1}^{K} z^{4 \ell+4 j} .
$$

The first sum in (48) converges to zero. The assumption $E \in(-2,2) \backslash\{0\}$ implies that $\left|z^{4}\right|=1$, $z^{4} \neq 1$ and therefore the second sum in 48 converges to zero as well. Thus asymptotically $\xi_{\ell, K}$ and $\zeta_{\ell, K}$ are independent standard real and complex normals. Collecting our estimates we formally get the SDE

$$
d X=\left(\begin{array}{cc}
i \lambda / 2 & 0 \\
0 & -i \lambda / 2
\end{array}\right) X d t+\frac{\sigma \rho}{2}\left(\begin{array}{cc}
i d \mathcal{B} & d \mathcal{W} \\
d \overline{\mathcal{W}} & -i d \mathcal{B}
\end{array}\right) X, \quad X(0)=I .
$$

from which Theorem 2 would follow after rescaling time and $\lambda$. In the case $E=0$, we get $\mathbb{E} \zeta_{\ell}^{2}=-1$, which implies that asymptotically $\xi_{\ell, K}$ and $\operatorname{Im} \zeta_{\ell, K}$ are independent standard normals and $\operatorname{Re} \zeta_{\ell, K}=$ 0 . In this case we formally get the SDE

$$
d X=\left(\begin{array}{cc}
i \lambda / 2 & 0 \\
0 & -i \lambda / 2
\end{array}\right) X d t+\frac{\sigma \rho}{2}\left(\begin{array}{cc}
i d \mathcal{B}_{1} & i d \mathcal{B}_{2} \\
-i d \mathcal{B}_{2} & -i d \mathcal{B}_{1}
\end{array}\right) X, \quad X(0)=I
$$

where $\mathcal{B}_{1}, \mathcal{B}_{2}$ are independent standard Brownian motions.

As we will show these computations can be made rigorous.

Proof of Theorem 2. In order to make the convergence argument precise, we use Proposition 26 which is a slight modification of Proposition 23 in Valkó and Virág (2009). We show the convergence in case of a single $\lambda \in \mathbb{C}$, the proof for finite dimensional marginals in $\lambda$ is very similar.

We will prove that $X_{\ell}^{n}=Z^{-1} Q_{\ell}^{n} Z$ converges to the solution of the SDE (49), from this the statement of the theorem follows. We can identify the $2 \times 2$ complex matrix $X_{\ell}^{n}$ with a vector in 
$\mathbb{R}^{8}$ by taking the real and imaginary parts of the entries. From 46 one gets that the conditional distribution of $X_{\ell+1}^{n}-X_{\ell}^{n}$ given $X_{\ell}^{n}=x$ is the same as that of

$$
Y_{\ell}^{n}(x):=\left(\frac{i \lambda}{2 n}-\frac{i \sigma \rho \omega_{\ell+1}}{2 \sqrt{n}}\right)\left(\begin{array}{cc}
1 & z^{2(\ell+1)} \\
-\bar{z}^{2(\ell+1)} & -1
\end{array}\right) x .
$$

From this $b^{n}(t, x)$ and $a^{n}(t, x)$ are computable. The function $b^{n}(t, x)$ will be a vector in $\mathbb{R}^{8}$ corresponding to the complex matrix

$$
\frac{i \lambda}{2}\left(\begin{array}{cc}
1 & z^{2(\ell+1)} \\
-\bar{z}^{2(\ell+1)} & -1
\end{array}\right) x \quad \text { with } \quad \ell=\lfloor n t\rfloor .
$$

The asymptotic variance $a^{n}(t, x)$ is a bit more cumbersome to write down, it is an $8 \times 8$ matrix with entries which are linear combinations of terms of the form of $A_{j} A_{k}, A_{j} \bar{A}_{k}$ and $\bar{A}_{j} \bar{A}_{k}$ with $j, k \in\{1,2\}^{2}$ where

$$
A=-\frac{i \sigma \rho}{2}\left(\begin{array}{cc}
1 & z^{2(\ell+1)} \\
-\bar{z}^{2(\ell+1)} & -1
\end{array}\right) x \quad \text { with } \quad \ell=\lfloor n t\rfloor .
$$

Clearly the coordinates of $a^{n}(t, x)$ are bilinear functions of $x$ and $\bar{x}$ with bounded coefficients (depending on $\sigma \rho$ and various powers of $z, \bar{z})$.

The functions $a(t, x), b(t, x)$ can be obtained from $a^{n}, b^{n}$ by writing zeros in place of the (nontrivial) powers of $z$ and $\bar{z}$, these are clearly $C^{2}$ functions. Condition 67 follows from the fact that $n^{-1} \sup _{\ell} \sum_{j=1}^{\ell} z^{2 j}$ and $n^{-1} \sup _{\ell} \sum_{j=1}^{\ell} z^{4 j}$ both converge to 0. Because of this in the integrals of (67) the $z$ terms will vanish in the limit and by the construction of $a$ and $b$ the other terms will cancel. Condition (68) is straightforward since $b, b^{n}$ are linear and $a, a^{n}$ are bilinear functions of $x, \bar{x}$ with bounded coefficients. The condition $(69)$ is a consequence of the assumption $\mathbb{E}\left|\omega_{\ell}\right|^{3}<\infty$ and since $X_{0}^{n}=I$ the last condition is also satisfied.

Thus we can apply Proposition 26 and the only thing left is to show that the functions $a(t, x)$, $b(t, x)$ correspond to the variance and drift functions corresponding to 49p. The fact that the drift function agrees is straightforward. To check the variance one needs to turn 49 into a real vector valued SDE which basically means that we need to take independent standard real and complex standard normals $B$ and $W$ and compute the variance of the random vector corresponding to

$$
-\frac{\sigma \rho}{2}\left(\begin{array}{cc}
i B & W \\
\bar{W} & -i B
\end{array}\right) x
$$

Using $\mathbb{E} B^{2}=\mathbb{E}|W|^{2}=1$ and $\mathbb{E} B W=\mathbb{E} W^{2}=0$ one can check that we get exactly $a(t, x)$ which finishes the proof of 49 . A time-change and the reparametrization $\lambda \rightarrow \lambda / \tau$ gives the SDE that 
is independent of $\sigma \rho$

$$
d X=\frac{1}{2}\left(\begin{array}{cc}
i \lambda & 0 \\
0 & -i \lambda
\end{array}\right) X d t+\frac{1}{2}\left(\begin{array}{cc}
i d \mathcal{B} & d \mathcal{W} \\
d \overline{\mathcal{W}} & -i d \mathcal{B}
\end{array}\right) X, \quad X(0)=I .
$$

and we get the claimed SDE through multiplication on the left by the matrix $Z$.

The same argument works for the proof of the first part of Theorem 7. The only difference is that in that case $z=i$ thus $z^{4 j}=\bar{z}^{4 j}=1$ and $a(t, x)$ will be defined accordingly.

\section{Convergence of the rescaled eigenvalue process}

In this section we prove the delocalization result (Theorem 11) and the point process limit theorems (Corollary 3 and 4).

\section{Tightness bounds}

Lemma 21. Let $\left(X_{k}(z): 1 \leq k \leq n, z \in \mathbb{C}\right)$ be random $d_{1} \times d_{2}$ matrices whose entries have finite second moments. Assume that $X_{k}(z)$ is analytic in $z$ and it is a martingale with respect to a filtration $\mathcal{F}_{k}$. Then for every $r_{1}<r_{2}<\infty$ and $t>0$

$$
\mathbb{P}\left\{\max _{1 \leq k \leq n,|z| \leq r_{1}} \operatorname{Tr} X_{k}(z) X_{k}(z)^{*} \geq t\right\} \leq t^{-1} \frac{r_{2}+r_{1}}{r_{2}-r_{1}} \frac{1}{2 \pi} \int_{-\pi}^{\pi} \mathbb{E} \operatorname{Tr} X_{n}\left(e^{i \theta r_{2}}\right) X_{n}\left(e^{i \theta r_{2}}\right)^{*} d \theta
$$

Proof. Since each entry $X_{k}(z)(i, j)$ is analytic in $z$, the Poisson formula and Jensen's inequality gives

$$
\left|X_{k}(z)(i, j)\right|^{2} \leq \frac{r_{2}+r_{1}}{r_{2}-r_{1}} \frac{1}{2 \pi} \int_{-\pi}^{\pi}\left|X_{k}\left(e^{i \theta r_{2}}\right)(i, j)\right|^{2} d \theta, \quad \text { for }|z| \leq r_{1} .
$$

Summing over all $i, j$ gives

$$
\operatorname{Tr} X_{k}(z) X_{k}(z)^{*} \leq \frac{r_{2}+r_{1}}{r_{2}-r_{1}} \frac{1}{2 \pi} x_{k}, \quad \text { for }|z| \leq r_{1},
$$

where

$$
x_{k}:=\int_{-\pi}^{\pi} \operatorname{Tr} X_{k}\left(e^{i \theta r_{2}}\right) X_{k}\left(e^{i \theta r_{2}}\right)^{*} d \theta
$$

As $x_{k}$ is a submartingale, the statement follows from Doob's inequality and Fubini's theorem.

Proof of Theorem 1. Let $\Xi_{\lambda}=\Xi=T\left(E+\frac{\lambda}{\rho n}\right)$. For large enough $n$ and all complex $\lambda,|\lambda| \leq r_{2}:=2 R$, the eigenvalues and eigenvectors of $\Xi$ are close to those of $T(E)$, we can write

$$
\Xi_{\lambda}=Z_{\lambda} D_{\lambda} Z_{\lambda}^{-1}
$$


with $\left\|Z_{\lambda}-Z\right\|_{2},\left\|D_{\lambda}-D\right\|_{2},\left\|Z_{\lambda}^{-1}-Z^{-1}\right\|_{2}$ all bounded by $c / n$ with $c$ depending on $E, R$. Since $T(E)$ has unit length eigenvalues we can find another constant $C=C(R, E)$ so that the eigenvalues of $\Xi^{k},|k| \leq n$ are uniformly bounded by $C$. Using this with the decomposition (52) we get that $\operatorname{Tr} \Xi^{k} \Xi^{* k}$ is also uniformly bounded for $|k| \leq n$.

Setting $S_{\ell}=\Xi^{-\ell} M_{\ell}$ we have, analogously to 45 , that

$$
S_{\ell}=S_{\ell-1}+\mathcal{E}_{\ell} S_{\ell-1}, \quad \mathcal{E}_{\ell}:=\Xi^{-\ell}\left(\begin{array}{cc}
0 & -\sigma n^{-1 / 2} \omega_{\ell} \\
0 & 0
\end{array}\right) \Xi^{\ell} .
$$

Since $\mathbb{E} \mathcal{E}_{\ell}=0$ we get

$$
\mathbb{E} S_{\ell} S_{\ell}^{*}=\mathbb{E} S_{\ell-1} S_{\ell-1}^{*}+\mathbb{E} \mathcal{E}_{\ell} S_{\ell-1} S_{\ell-1}^{*} \mathcal{E}_{\ell}^{*}
$$

Taking the trace and conditioning on $S_{\ell-1}$ we get

$$
\begin{aligned}
\operatorname{Tr} \mathbb{E} S_{\ell} S_{\ell}^{*}-\operatorname{Tr} \mathbb{E} S_{\ell-1} S_{\ell-1}^{*} & =\mathbb{E} \operatorname{Tr} \mathcal{E}_{\ell} S_{\ell-1} S_{\ell-1}^{*} \mathcal{E}_{\ell}^{*}=\mathbb{E} \operatorname{Tr} S_{\ell-1} S_{\ell-1}^{*} \mathcal{E}_{\ell}^{*} \mathcal{E}_{\ell} \\
& =\operatorname{Tr}\left(\mathbb{E} S_{\ell-1} S_{\ell-1}^{*}\right)\left(\mathbb{E} \mathcal{E}_{\ell}^{*} \mathcal{E}_{\ell}\right) \leq \operatorname{Tr}\left(\mathbb{E} S_{\ell-1} S_{\ell-1}^{*}\right) \operatorname{Tr}\left(\mathbb{E} \mathcal{E}_{\ell}^{*} \mathcal{E}_{\ell}\right)
\end{aligned}
$$

In the last step we used that if $A, B$ are positive semidefinite matrices of the same dimension then $\operatorname{Tr} A B \leq \operatorname{Tr} A \operatorname{Tr} B$. Using the 52 and the bounds on $Z_{\lambda}, D_{\lambda}$ one gets that $\operatorname{Tr}\left(\mathbb{E} \mathcal{E}_{\ell}^{*} \mathcal{E}_{\ell}\right) \leq c \sigma^{2} / n$ and it follows that for all $n \geq n_{0}, 0 \leq \ell \leq n$ and $\lambda \in \mathbb{C}$ with $|\lambda| \leq r_{2}$ we have

$$
\mathbb{E}\left[\operatorname{Tr}\left(S_{\ell}^{*} S_{\ell}\right)\right] \leq C_{1}
$$

Note that $\left(S_{\ell}: 0 \leq \ell \leq n\right)$ is martingale analytic in the parameter $\lambda$. Then Lemma 21 implies that (9) holds for $S_{\ell}^{\lambda}$ in instead of $M_{\ell}^{\lambda}$ with probability $1-c / t$. To translate the result for $M_{\ell}^{\lambda}$ we use the estimate

$$
\operatorname{Tr} M_{\ell} M_{\ell}^{*}=\operatorname{Tr} \Xi^{* \ell} \Xi^{\ell} S_{\ell} S_{\ell}^{*} \leq \operatorname{Tr} \Xi^{* \ell} \Xi^{\ell} \operatorname{Tr} S_{\ell} S_{\ell}^{*} \leq C \operatorname{Tr} S_{\ell} S_{\ell}^{*}
$$

To prove the second part of the theorem it is enough to show that if we assume that $\operatorname{Tr} M_{\ell} M_{\ell}^{*}$ is bounded by $t$ uniformly in $\ell$ and $\lambda$ then (10) holds. Let $\psi$ be a normalized eigenvector of $H_{n}$ corresponding to the eigenvalue $e=E+\frac{\lambda}{\rho n} \in\left[E-\frac{R}{\rho n}, E+\frac{R}{\rho n}\right]$ and let $\Psi_{\ell}=\left(\begin{array}{c}\psi_{\ell+1} \\ \psi_{\ell}\end{array}\right)$. For each $0 \leq k, \ell \leq n$, the transfer matrix description of the eigenvalue equation gives $\Psi_{k}=M_{k} \Psi_{0}=$ $M_{k}\left(M_{\ell}\right)^{-1} \Psi_{\ell}, \psi_{0}=\psi_{n+1}=0$. Since for the induced operator norm $\|\cdot\|_{2,2}$ we have

$$
\|A\|_{2,2}=\sqrt{\lambda_{\max }\left(A A^{*}\right)} \leq \sqrt{\operatorname{Tr} A A^{*}}
$$

we get the bound $\left\|M_{\ell}\right\|_{2,2}<\sqrt{t}$. But $M_{\ell}$ is a $2 \times 2$ matrix and $\operatorname{det} M_{\ell}=1$ so $\left\|M_{\ell}\right\|_{2,2}=\left\|M_{\ell}^{-1}\right\|_{2,2}$. This leads to $\left\|M_{\ell}^{-1}\right\|_{2,2}<\sqrt{t}$ and

$$
\left\|\Psi_{k}\right\|_{2}^{2} \leq\left\|M_{k}\right\|_{2,2}^{2}\left\|M_{\ell}^{-1}\right\|_{2,2}^{2}\left\|\Psi_{\ell}\right\|_{2}^{2}<t^{2}\left\|\Psi_{\ell}\right\|_{2}^{2}
$$

Summing the last inequality over all $0 \leq k \leq n$ gives $2<(n+1) t^{2}\left\|\Psi_{\ell}\right\|^{2}$ and summing over all $0 \leq \ell \leq n$ gives $(n+1)\left\|\Psi_{k}\right\|^{2}<2 t^{2}$. 


\section{Proof of Corollary 3}

By (6) and (11) for each $n$, the rescaled eigenvalues $\lambda_{k}$ are given by the zeros of the random analytic function $g_{n}: \mathbb{C} \rightarrow \mathbb{C}$ :

$$
g_{n}(\lambda):=\operatorname{det}\left(Q_{n}^{\lambda}\left(\begin{array}{l}
1 \\
0
\end{array}\right), B_{n}\right), \quad B_{n}:=T^{-n}\left(\begin{array}{l}
0 \\
1
\end{array}\right) .
$$

Our assumption is that along a subsequence $n_{j}, B_{n}$ converges to a vector $B \in \mathbb{R}^{2}$. It follows from Theorem 2 that for any fixed $\left(\lambda_{1}, \cdots, \lambda_{m}\right) \in \mathbb{C}^{m}$, the random vector $\left(g_{n_{j}}\left(\lambda_{1}\right), \cdots, g_{n_{j}}\left(\lambda_{m}\right)\right) \in \mathbb{C}^{m}$ converges in distribution to a random vector

$$
\left(g\left(\lambda_{1}\right), \cdots, g\left(\lambda_{m}\right)\right):=\left(\operatorname{det}\left(Q^{\lambda_{1} / \tau}(\tau)\left(\begin{array}{l}
1 \\
0
\end{array}\right), B\right), \cdots, \operatorname{det}\left(Q^{\lambda_{m} / \tau}(\tau)\left(\begin{array}{l}
1 \\
0
\end{array}\right), B\right)\right)
$$

where $\left(Q^{\lambda_{1} / \tau}(t), \cdots Q^{\lambda_{m} / \tau}(t)\right)$ is the solution to the SDE (13). We need to show that the family of distributions in (55), indexed by $\left(\lambda_{1}, \cdots, \lambda_{m}\right) \in \mathbb{C}^{m}$, defines a random analytic function $g(\lambda)$ and that the mode of convergence $g_{n_{j}}(\lambda) \rightarrow g(\lambda)$ is strong enough to ensure convergence of zeros.

We will use the following notions of convergence. Let $\mathcal{A}\left(D, \mathbb{R}^{d}\right)$ denote the space of analytic functions from a connected open set $D$ in $\mathbb{C}$ to $\mathbb{C}^{d}$. We equip $\mathcal{A}\left(D, \mathbb{R}^{d}\right)$ with the metric

$$
d(f, g):=\sum_{r=1}^{\infty} 2^{-r} \frac{\|f-g\|_{r}}{1+\|f-g\|_{r}}, \quad \text { where } \quad\|h\|_{r}:=\max _{z \in D \cap\{|z|<r\}}\|h(z)\| .
$$

Then $\left(\mathcal{A}\left(D, \mathbb{R}^{d}\right), d\right)$ is a complete separable metric space and convergence in $d$ is the local uniform convergence. A random analytic function in $\mathcal{A}\left(D, \mathbb{R}^{d}\right)$ is a measurable mapping $\omega \rightarrow f^{\omega}$ from a probability space $(\Omega, \mathcal{F}, P)$ to $\left(\mathcal{A}\left(D, \mathbb{R}^{d}\right), \mathcal{B}\right)$, where $\mathcal{B}$ is the Borel $\sigma$-field generated by the metric $d$. The law of $f$ is the induced probability measure $\rho_{f}$ on $\left(\mathcal{A}\left(D, \mathbb{R}^{d}\right), \mathcal{B}_{d}\right)$. A sequence $f_{\ell}^{\omega}$ of random analytic functions is said to converge in law to a random analytic function $f^{\omega}$ if $\rho_{f_{\ell}} \rightarrow \rho_{f}$ in the usual sense of weak convergence.

Proposition 22. Suppose

(1) $f_{\ell}^{\omega}$ is a sequence of random analytic functions in $\mathcal{A}\left(D, \mathbb{R}^{d}\right)$ such that for every $0<r<\infty$,

$$
\lim _{C \rightarrow \infty} \mathbb{P}\left\{\sup _{\ell \geq 1,|\lambda| \leq r}\left|f_{\ell}(\lambda)\right|>C\right\}=0 .
$$

(2) for each $m \geq 1$ and $\widetilde{\lambda}=\left(\lambda_{1}, \lambda_{2}, \cdots, \lambda_{m}\right) \in \mathbb{C}^{m}$ there is a probability distribution $\nu^{\tilde{\lambda}}$ on $\mathbb{C}^{m}$ and the random vector $\left(f_{\ell}^{\omega}\left(\lambda_{1}\right), f_{\ell}^{\omega}\left(\lambda_{2}\right), \cdots, f_{\ell}^{\omega}\left(\lambda_{m}\right)\right) \in \mathbb{C}^{m}$ converges in law to $\nu^{\tilde{\lambda}}$.

Then there is a random analytic function $f^{\omega}$ in $\mathcal{A}\left(D, \mathbb{R}^{d}\right)$ such that $f_{\ell}^{\omega}$ converges in law to $f^{\omega}$. Moreover for each $\widetilde{\lambda}=\left(\lambda_{1}, \lambda_{2}, \cdots, \lambda_{m}\right) \in \mathbb{C}^{m},\left(f^{\omega}\left(\lambda_{1}\right), f^{\omega}\left(\lambda_{2}\right), \cdots, f^{\omega}\left(\lambda_{m}\right)\right) \in \mathbb{C}^{m}$ has distribution $\nu^{\tilde{\lambda}}$. 
Proof. For each disk $D_{r}:=\{\lambda \in \mathbb{C}:|\lambda|<r\}$, the bound in (56) together with Montel's and Prokhorov's theorems imply that a subsequence of $f_{\ell}$ restricted to $D_{r}$ converges in law to a random analytic function $f_{r}$ on $D_{r}$. Then by a diagonal argument, there is a subsequence of $f_{\ell_{k}}$ such that for each integer $r$, the restriction of $f_{\ell_{k}}$ to $D_{r}$ converges to to random analytic function $f_{r}$ on $D_{r}$. The distributions of the functions $f_{r}$ are consistent with respect to restricting to smaller discs, and thus there is a random analytic function $f$ on $\mathbb{C}$ such that $f_{\ell_{k}} \rightarrow f$ in law. Condition (2) is strong enough to ensure that $f$ is unique and thus $f_{\ell} \rightarrow f$.

Let $\mathcal{A}=\mathcal{A}(\mathbb{C}, \mathbb{C})$ and $\mathcal{A}_{0}:=\mathcal{A} \backslash\{0\}$, i.e. we discard the identically zero function. $\mathcal{M}$ denotes the set of nonnegative Borel measures on $\mathbb{C}$, that are finite on bounded subsets of $\mathbb{C}$. We consider the local weak topology on $\mathcal{M}$ : a sequence $\mu_{\ell} \in \mathcal{M}$ is said to converge to $\mu \in \mathbb{C}$ if for every continuous function $\psi: \mathbb{C} \rightarrow \mathbb{R}$ of compact support, $\int \psi d \mu_{\ell} \rightarrow \int \psi d \mu$. For $f \in \mathcal{A}_{0}$, we denote by

$\mu_{f}$ the zero counting measure of $f$, i.e. $\mu_{f}=\sum_{f(z)=0} m(z) \delta(z)$, where $m(z)$ is the multiplicity of the zero $z$. As an elementary consequence of Cauchy's integral formula, we have that for $f_{\ell}, f \in \mathcal{A}_{0}$, $d\left(f_{\ell}, f\right) \rightarrow 0$ implies $\mu_{f_{\ell}} \rightarrow \mu_{f}$.

A random measure in $\mathcal{M}$ is a measurable function $\omega \rightarrow \mu^{\omega}$ to $\mathcal{M}$ (with the Borel $\sigma$-algebra).

If $f^{\omega}$ is a random analytic function in $\mathcal{A}$ with $\mathbb{P}(f \equiv 0)=0$, then $\mu_{f}$ is a random measure in $\mathcal{M}$. If $f_{\ell}^{\omega}$ converges in law to $f^{\omega}$ and $\mathbb{P}\left(f_{\ell} \equiv 0\right)=\mathbb{P}(f \equiv 0)=0$, then the corresponding random measure $\mu_{f_{\ell}^{\omega}}$ converges in law to $\mu_{f \omega}$.

We can now complete the proof of Corollary 3. The appropriate part of Theorem 7 can be proved the same way.

Proof of Corollary 3. The tightness bound (9), Theorem 2 and Proposition 22 guarantee that the random analytic function $\mathbb{C} \ni \lambda \rightarrow Q_{n}^{\lambda} \in M_{2}(\mathbb{C})$ converges in distribution to the random analytic function $\mathbb{C} \ni \lambda \rightarrow Q^{\lambda / \tau}(\tau) \in M_{2}(\mathbb{C})$ as $n \rightarrow \infty$. Then the random analytic function $g_{n_{j}}(\lambda)$ defined in (54) converges to the random analytic function $g(\lambda)$ defined in (55). In addition it is easy to see that $\mathbb{P}\left(g_{n} \equiv 0\right)=\mathbb{P}(g \equiv 0)=0$. Thus $\mu_{g_{n_{j}}^{\omega}}$ converges in law to $\mu_{g^{\omega}}$.

\section{The phase function}

Proof of Corollary 4. The existence and uniqueness of the analytic solution of (15), as well as the monotonicity of $\varphi^{\lambda}(\tau)$ will be shown in Section 7 .

To prove the second part of the theorem we will first assume that $z^{n_{j}+1}$ converges to $e^{i \theta}$. Then $T^{n_{j}}(E)$ converges to a matrix $\tilde{T}$ and

$$
\tilde{T} Z=\lim T^{n_{j}}(E) Z=Z \lim \left(\begin{array}{cc}
\bar{z}^{n_{j}} & 0 \\
0 & z^{n_{j}}
\end{array}\right)=\left(\begin{array}{cc}
\bar{z} & z \\
1 & 1
\end{array}\right)\left(\begin{array}{cc}
z e^{-i \theta} & 0 \\
0 & \bar{z} e^{i \theta}
\end{array}\right)=\left(\begin{array}{cc}
e^{-i \theta} & e^{i \theta} \\
z e^{-i \theta} & \bar{z} e^{i \theta}
\end{array}\right) .
$$


By Corollary 3 we need to identify the zeros of

$$
\left[\tilde{T} Q^{\lambda}(\tau)\right]_{11}=\left[\tilde{T} Z \tilde{X}^{\lambda}(\tau) Z^{-1}\right]_{11}=\left[\tilde{T} Z X^{\lambda}(\tau)\right]_{11}
$$

where $\tilde{X}^{\lambda}$ satisfies the SDE 51 . By linearity, $X:=\tilde{X} Z^{-1}$ satisfies the same SDE, but with initial condition $X^{\lambda}(0)=Z^{-1}$.

Note that if $\lambda \in \mathbb{R}$ then $X^{\lambda}$ is a matrix of the form $\left(\begin{array}{cc}a & b \\ \bar{a} & \bar{b}\end{array}\right)$. Indeed, this holds for $t=0$ and it is preserved by the evolution by (49). So we have

$$
\left[\tilde{T} Z X^{\lambda}(\tau)\right]_{11}=e^{-i \theta} X^{\lambda}(\tau)_{11}+e^{i \theta} X^{\lambda}(\tau)_{21}=2 \operatorname{Re}\left[e^{-i \theta} X^{\lambda}(\tau)_{11}\right] .
$$

We rewrite the SDE's for the matrix entries as follows:

$$
\begin{array}{ll}
2 d X_{11}=i \lambda X_{11} d t+i X_{11} d \mathcal{B}+\bar{X}_{11} d \mathcal{W}, & X_{11}(0)=i \rho / 2 \\
2 d X_{12}=i \lambda X_{12} d t+i X_{12} d \mathcal{B}+\bar{X}_{12} d \mathcal{W}, & X_{12}(0)=i z \rho / 2
\end{array}
$$

Itô's formula gives

$$
d \operatorname{det} \tilde{X}^{\lambda}=0 \quad \text { and } \quad 2 i \operatorname{Im}\left[X_{11} \bar{X}_{12}\right]=\operatorname{det} \tilde{X}^{\lambda}(t)=\operatorname{det} Z^{-1}=i \rho / 2
$$

which shows that $X_{11}^{\lambda}(t)$ is never equal to 0 , and the phase function $\varphi^{\lambda}(t)$ is well-defined via

$$
e^{i \varphi^{\lambda}(t)}=\frac{i X_{11}^{\lambda}(t)}{\overline{i X_{11}^{\lambda}(t)}}, \quad \varphi^{\lambda}(0)=0 .
$$

Itô's formula applied to (58) shows that $\varphi$ satisfies 150 with $-d \mathcal{W}$ in place of $d \mathcal{W}$. Also, the zeros of (57) are given by the solutions of $\operatorname{Re}\left[e^{-i \theta-i \pi / 2} i X^{\lambda}(\tau)_{11}\right]=0$, or, equivalently, $-\theta-\pi / 2+\varphi^{\lambda}(\tau) / 2 \in$ $\pi \mathbb{Z}$.

So far we have shown that if $z^{n_{j}+1} \rightarrow e^{i \theta}$ then

$$
\Lambda_{n_{j}} \Rightarrow\left\{\lambda: \varphi^{\lambda / \tau}(\tau) \in 2 \theta+\pi+2 \pi \mathbb{Z}\right\} \stackrel{d}{=} \operatorname{Sch}_{\tau}+2 \theta+\pi
$$

where the last equality follows by the definition (16) and Lemma 18. It follows that

$$
\Lambda_{n}-2 \arg \left(z^{n+1}\right)-\pi \Rightarrow \operatorname{Sch}_{\tau} .
$$

Since $2 \arg \left(z^{n+1}\right)-\arg \left(z^{2 n+2}\right)$ is either 0 or $2 \pi$ and $\operatorname{Sch}_{\tau} \stackrel{d}{=} \operatorname{Sch}_{\tau}+2 \pi$ the statement of the corollary follows. 


\section{The limit theorem for the the decaying model}

In this section we discuss Theorems 14 and 15 . The proof of Theorem 14 can be done exactly the same way as that of Theorem 2. Since we only need to prove the convergence in an interval $[0,1-\varepsilon]$ for a given $\varepsilon>0$, the fact that the coefficient of the noise term blows up at $t=1$ will not cause any problems.

Theorem 15 can be proved the way 26) was derived for the $\beta$-Hermite ensemble in Valkó and Virág (2009). The proof that we present below is not fully self-contained, we only highlight the main points of the arguments.

Proof of Theorem 15. As an analogue of the continuous time phase function we define the discrete phase function $\varphi_{\ell}^{\lambda}$ with the identity $e^{i \varphi_{\ell}^{\lambda}}=X_{\ell}^{\lambda}[1,1] / X_{\ell}^{\lambda}[2,1]$ and the relative phase function $\alpha_{\ell}^{\lambda}$ as $\varphi_{\ell}^{\lambda}-\varphi_{\ell}^{0}$. Note that $\varphi_{\ell}^{\lambda}$ can be defined as a continuous function in $\lambda$ for any fixed $n$ which will make $\alpha_{\ell}^{\lambda}$ a well defined function. Equation 46 can be converted to a recursion for $e^{i \varphi_{\ell}^{\lambda}}$ :

$$
e^{i \varphi_{\ell}^{\lambda}}=\mathcal{T}_{\ell}^{\lambda}\left(e^{i \varphi_{\ell-1}^{\lambda}}\right)
$$

where

$$
\mathcal{T}_{\ell}^{\lambda}(v)=z^{2 \ell} \mathcal{X}_{\ell}^{\lambda}\left(z^{-2 \ell} v\right), \quad \mathcal{X}_{\ell}^{\lambda}(\xi)=\frac{\xi\left(1+i \rho \varepsilon_{\ell} / 2\right)+i \rho \varepsilon_{\ell} / 2}{1-i \rho \varepsilon_{\ell} / 2-i \rho \varepsilon_{\ell} \xi / 2} .
$$

$\lambda$ is an eigenvalue if

$$
X_{n}^{\lambda}\left(\begin{array}{l}
1 \\
0
\end{array}\right)=c Z^{-1} T^{-n}\left(\begin{array}{l}
0 \\
1
\end{array}\right)=\frac{c i \rho}{2}\left(\begin{array}{c}
-z^{n+1} \\
\bar{z}^{n+1}
\end{array}\right)
$$

which is equivalent to $e^{i \varphi_{n}^{\lambda}}=-z^{2 n+2}$. The discrete version of the Sturm-Liouville theory implies that the number of eigenvalues in a given interval $\left[\lambda_{1}, \lambda_{2}\right]$ is given by the number of solutions of $e^{i x}=-z^{2 n+2}$ with $x \in\left[e^{i \varphi_{n}^{\lambda_{1}}}, e^{i \varphi_{n}^{\lambda_{2}}}\right]$. We can also count the eigenvalues using intermediate values of the phase function $\varphi^{\lambda}$. We define $\widetilde{\varphi}_{k}^{\lambda}$ as a continuous function in $\lambda$ recursively using

$$
e^{i \widetilde{\varphi}_{0}^{\lambda}}=-z^{2 n+2}, \quad e^{i \widetilde{\varphi}_{k+1}^{\lambda}}=\left[\mathcal{T}_{n-k}^{\lambda}\right]^{-1}\left(e^{i \widetilde{\varphi}_{k}^{\lambda}}\right)
$$

Then the number of eigenvalues in a given interval $\left[\lambda_{1}, \lambda_{2}\right]$ is given by

$$
\#\left(\left[\left(\varphi_{n-k}^{\lambda_{1}}-\widetilde{\varphi}_{k}^{\lambda_{1}}\right),\left(\varphi_{n-k}^{\lambda_{2}}-\widetilde{\varphi}_{k}^{\lambda_{2}}\right)\right] \cap 2 \pi \mathbb{Z}\right)
$$

The main steps of the theorem are as follows. The first step is straightforward from Theorem 14 .

Step 1. For every $0<\varepsilon<1$ we have $\alpha_{\lfloor n(1-\varepsilon\rfloor)}^{\lambda} \Rightarrow \alpha^{\lambda}(1-\varepsilon)$ in the sense of finite dimensional distributions where $\alpha^{\lambda}(t)$ is the solution of SDE (25).

The next step shows that the relative phase function $\alpha^{\lambda}$ cannot change too much from $n(1-\varepsilon)$ to $n-k$. 
Step 2. There exist a constant $c>0$ depending only on $\sigma, \rho$ and $\Lambda$ so that for every $|\lambda| \leq \Lambda$ and $k \leq \varepsilon n$ we have

$$
\mathbb{E}\left[\left(\alpha_{\lfloor n(1-\varepsilon\rfloor)}^{\lambda}, \alpha_{n-k}^{\lambda}\right) \wedge 1\right] \leq c\left(\mathbb{E} \operatorname{dist}\left(\alpha_{\lfloor n(1-\varepsilon\rfloor)}^{\lambda}, 2 \pi \mathbb{Z}\right)+\varepsilon^{1 / 2}+n^{-1 / 2}+k^{-1}\right)
$$

The proof of Step 2 can be done in a similar way as in Valkó and Virág (2009). By analyzing the recursion 60 we can get a precise estimate on $\mathbb{E}\left(\alpha_{\ell+1}^{\lambda} \mid \varphi_{\ell}^{\lambda}, \varphi_{\ell}^{0}\right)$. This can be turned into a Gronwall type estimate for $\operatorname{dist}\left(\alpha_{\ell}^{\lambda}, \pi \mathbb{Z}\right)$ which leads to 63 . (See Sections 6.1 and 6.2 in Valkó and Virág (2009) for details.) In order to estimate certain error terms one can take advantage of the fact that the rotation $z^{2 \ell}$ in 61 has an averaging effect:

$$
\left|\sum_{\ell=\ell_{1}}^{\ell_{2}} z^{2 \ell} a_{\ell}\right| \leq C\left(\left|a_{\ell_{1}}\right|+\sum_{\ell=\ell_{1}}^{\ell_{2}-1}\left|a_{\ell+1}-a_{\ell}\right|\right)
$$

We would like to note that this makes our case a lot easier to deal with than the one in Valkó and Virág (2009) where the dependence of the oscillation on $\ell$ was more complicated and needed much more involved estimates using harmonic analytic tools.

The next step shows that asymptotically in the formula 62 only $\alpha_{n-k}^{\lambda}$ 'matters'. The proof is analogue to the one presented in Sections 6.3 and 6.4 in Valkó and Virág (2009).

Step 3. If $k=k(n) \rightarrow \infty$ with $k / n \rightarrow 0$ then $\varphi_{n-k}^{0}$ converges to a uniform random variable on $[0,2 \pi]$ modulo $2 \pi$ in distribution. If $k$ is fixed then $\tilde{\varphi}_{k}^{\lambda}-\tilde{\varphi}_{k}^{0} \rightarrow 0$ in probability.

Now we have all the ingredients for the proof. Suppose that we want to show that for a given vector $\left(\lambda_{1}, \ldots, \lambda_{d}\right)$ we have

$$
\text { the number of ev's in }\left[0, \lambda_{1}\right],\left[0, \lambda_{2}\right], \ldots,\left[0, \lambda_{d}\right] \Rightarrow\left(\alpha^{\lambda_{1}}(1), \ldots, \alpha^{\lambda_{d}}(1)\right) \text {. }
$$

By the previous statements we can find an appropriate sequence $k=k(n) \rightarrow \infty$ so that

$$
\left(\alpha_{n-k}^{\lambda_{i}}, i=1, \ldots, d\right) \Rightarrow\left(\alpha^{\lambda_{i}}(1), i=1, \ldots, d\right)
$$

and $\tilde{\varphi}_{k}^{\lambda_{i}}-\tilde{\varphi}_{k}^{0} \rightarrow 0$ in probability for $i=1, \ldots, d$. This means that if we apply formula 62 with $\lambda_{1}=0, \lambda_{2}=\lambda_{i}$ then the the length of the interval will converge to $\alpha^{\lambda_{i}}(1) \in 2 \pi \mathbb{Z}$ and the endpoint will become uniform modulo $2 \pi$. Hence the number of lattice points in the $i^{\text {th }}$ interval will converge to $\frac{1}{2 \pi} \alpha^{\lambda_{i}}(1)$ which proves the theorem along the found subsequence. But the argument can be repeated to find a converging sub-subsequence of any subsequence, and since we always get the same limit this shows the weak convergence along the original (full) sequence as well. 


\section{Appendix}

The appendix contains the proof for the existence of unique analytic solutions for the discussed SDEs and a technical proposition about the convergence of discrete time Markov chains to stochastic differential equations.

\section{Uniqueness and analyticity of the limiting SDE's}

Proposition 23. The stochastic differential equations (13), (15), (17), (18), (23), (24) all have unique strong solutions which are analytic in $\lambda$. Moreover the solutions of (15), (18) and (24) are strictly increasing in $\lambda$ for any positive $t$.

Proof. The coefficients of these SDE's are all uniformly Lipschitz so they have unique strong solutions for any finite vector $\underline{\lambda} \in \mathbb{C}^{d}$. (Note that in the decaying case one may assume $t \in[0,1-\varepsilon]$.)

In order to show that one can realize these solutions for all values of $\lambda$ together in a way that the dependence on $\lambda$ is analytic requires some extra work.

One possibility to deal with this problem is to use the smooth dependence of the solution of an SDE on the initial condition. We will use the following theorem which is a slight modification of Theorem 40 in Protter (2005).

Theorem 24 (Protter, Theorem 40). Let $f_{\alpha}^{i}: \mathbb{R}^{d} \rightarrow \mathbb{R}, 1 \leq i \leq d, 0 \leq \alpha \leq m$ be functions with locally Lipschitz derivatives up to order $N$ for some $0 \leq N \leq \infty$. Then there exists a solution $X(t, \omega, x)$ to

$$
X_{t}^{i}=x_{i}+\int_{0}^{t} f_{0}^{i}\left(X_{s}\right) d s+\sum_{\alpha=1}^{m} \int_{0}^{t} f_{\alpha}^{i}\left(X_{s}\right) d B_{s}^{\alpha}, \quad i=1, \ldots, d
$$

which is $N$ times continuously differentiable in the open set $\{x: \zeta(x, \omega)>t\}$ where $\zeta$ is the explosion time of the solution. Moreover the respective derivatives in $x$ will satisfy the formal derivative of equation 64.

We can encode the dependence on $\lambda$ in (13) into dependence on initial condition by introducing extra variables for $\operatorname{Re} \lambda, \operatorname{Im} \lambda$ and the extra equations $d \operatorname{Re} \lambda=0, d \operatorname{Im} \lambda=0$. Since for any fixed $\lambda$ the SDE has globally Lipschitz coefficients we will have $\zeta=\infty$. This shows that there exists a solution to (13) which is twice differentiable in the real variables $(x, y)=(\operatorname{Re} \lambda, \operatorname{Im} \lambda)$. The fact that we also get analyticity in $\lambda \in \mathbb{C}$ follows from the fact that the Cauchy-Riemann equations are satisfied. Indeed, at time $t=0$ we have $\partial_{x} X(t)=i \partial_{y} X(t)$ and it can be checked that the two processes satisfy the same SDE which means that the previous equation is preserved.

The same proof works for (15), (17), (18). In the case of (23) and (24) the coefficients depend on $t$ as well, but introducing an extra variable for $t$ takes care of this (note that in this case $t \in[0,1)$ ). 
To prove that the solution $\varphi^{\lambda}(t)$ of 15$)$ is increasing in $\lambda \in \mathbb{R}$ we first compute the SDE for its derivative.

$$
d\left(\partial_{\lambda} \varphi^{\lambda}(t)\right)=d t+\operatorname{Re}\left[-i \partial_{\lambda} \varphi^{\lambda}(t) e^{-i \varphi^{\lambda}(t)} d \mathcal{W}\right]=d t+\partial_{\lambda} \varphi^{\lambda}(t) \operatorname{Im}\left[e^{-i \varphi^{\lambda}(t)} d \mathcal{W}\right], \quad \partial_{\lambda} \varphi^{\lambda}(0)=0
$$

For a given $\lambda$ the derivative solves the SDE

$$
d a=d t+\frac{1}{\sqrt{2}} a d \mathcal{B}_{\lambda}, \quad a(0)=0
$$

with $d \mathcal{B}_{\lambda}=\sqrt{2} \operatorname{Im}\left[e^{-i \varphi^{\lambda}(t)}, d \mathcal{W}\right]$ and a simple coupling argument shows that this is always positive for $t>0$. (Actually, in this case one can even solve the SDE explicitly.) Similar proof works for (18) and (24). Note that using the carousel representation of the Section 3 one can also prove the monotonicity for 15 and (24).

Corollary 25. For a given $\lambda \in \mathbb{R}$ the distribution of $\partial_{\lambda} \varphi^{\lambda}(t)$ is the same as

$$
\int_{0}^{t} e^{-\frac{1}{\sqrt{2}}\left(\mathcal{B}_{s}-\mathcal{B}_{t}\right)+\frac{1}{4}(s-t)} d s=e^{\frac{1}{\sqrt{2}} \mathcal{B}_{t}-\frac{1}{4} t} \int_{0}^{t} e^{-\frac{1}{\sqrt{2}} \mathcal{B}_{s}+\frac{1}{4} s} d s
$$

Proof. Using Itô's formula it is straightforward to check that the process given in the statement of the corollary satisfies the SDE (66).

\section{Convergence of discrete time Markov processes to SDEs}

Proposition 26. Fix $T>0$ and for each $n \geq 1$ consider a Markov chain

$$
\left(X_{\ell}^{n} \in \mathbb{R}^{d}, \ell=0 \ldots\lfloor n T\rfloor\right)
$$

with $\mathbb{E}\left\|X_{\ell}^{n}\right\|^{2}<\infty$. For $x \in \mathbb{R}^{d}$, let $Y_{\ell}^{n}(x) \in \mathbb{R}^{d}$ be distributed as the increment of $X_{\ell+1}^{n}-X_{\ell}^{n}$ given $X_{\ell}^{n}=x$. For $0 \leq t \leq T$ and $x \in \mathbb{R}^{d}$, let $b^{n}(t, x) \in \mathbb{R}^{d}$ and $a^{n}(t, x) \in M_{d}^{\mathrm{sym}}(\mathbb{R})$ be defined by

$$
b^{n}(t, x):=n \mathbb{E} Y_{\lfloor n t\rfloor}^{n}(x), \quad a^{n}(t, x):=n \mathbb{E} Y_{\lfloor n t\rfloor}^{n}(x) Y_{\lfloor n T\rfloor}^{n}(x)^{\mathrm{T}} .
$$

We make the following assumptions.

(1) There are $C^{2}$ functions $a:[0, T] \times \mathbb{R}^{d} \rightarrow M_{d}^{\mathrm{sym}}(\mathbb{R})$ and $b:[0, T] \times \mathbb{R}^{d} \rightarrow M_{d}(\mathbb{R})$ such that for every $R<\infty$,

$$
\sup _{0 \leq t \leq T,|x| \leq R}\left\|\int_{0}^{t}\left(a^{n}(s, x)-a(s, x)\right) d s\right\|+\sup _{0 \leq t \leq T,|x| \leq R}\left\|\int_{0}^{t}\left(b^{n}(s, x)-b(s, x)\right) d s\right\| \rightarrow 0 .
$$

(2) For every $R<\infty$ there is a constant $c_{R}<\infty$ such that

$$
\left\|a^{n}(t, x)-a^{n}(t, y)\right\|+\left\|b^{n}(t, x)-b^{n}(t, y)\right\| \leq c_{R}\|x-y\|,
$$


for all $n \geq 1, t \in[0, T],\|x\| \leq R$ and $\|y\| \leq R$. The same inequality holds for $a$ and $b$.

(3) For every $R<\infty$ there is a constant $d_{R}<\infty$ such that

$$
\sup _{0 \leq \ell \leq n,\|x\| \leq R} \mathbb{E}\left[\left\|Y_{\ell}^{n}(x)\right\|^{3}\right] \leq d_{R} n^{-3 / 2}
$$

(4) The initial condition $X_{0}^{n}$ converges in distribution to $X_{0}$ with $\mathbb{E}\left\|X_{0}\right\|^{2}<\infty$.

Then $\left(X_{\lfloor n T\rfloor}^{n}, 0 \leq t \leq T\right)$ converges weakly in $D[0, T]$ to the unique solution of the SDE

$$
d X(t)=b(t, X(t)) d t+g(t, X(t)) d \mathcal{B}(t), \quad X(0)=X_{0},
$$

where $\mathcal{B}(t)$ is the d-dimensional Brownian motion and $g:[0, T] \times \mathbb{R}^{d} \rightarrow M_{d}(\mathbb{R})$ is any $C^{2}$ function with

$$
g(t, x) g(t, x)^{\mathrm{T}}=a(t, x) .
$$

Note: one can always take $g(t, x):=(a(t, x))^{1 / 2}$ but it can be useful to make other choices for which $a(t, x)$ has sparser structure than $(a(t, x))^{1 / 2}$ and the resulting SDE has a simpler noise term.

Proof. This is Proposition 23 in Valkó and Virág (2009) with two small changes: there the supremum is for all $x$ in (1) and (3) and the functions $a, b$ are assumed to have bounded derivatives instead of being Lipschitz in $x$. The proof is very similar, but we include it for the sake of completeness.

Let $\|\cdot\|_{\infty}$ denote supremum norm on $[0, T]$. For a two-parameter function $f$ and $x \in \mathbb{R}$ let $\mathcal{I}$ denote the integral $\mathcal{I}_{f, x}(t)=\int_{0}^{t} f(s, x) d s$. We recycle this notation for a function $X:[0, T] \rightarrow \mathbb{R}$ to write $\mathcal{I}_{f, X}(t)=\int_{0}^{t} f(s, X(s)) d s$.

Because of our assumptions on $a$ and $b$ the well-posedness of the martingale problem follows from Theorem 5.3.7 of Ethier and Kurtz (1986) (see especially the remarks following the proof), and even pathwise uniqueness holds. This means that $(70)$ has a solution $X$ with initial condition $X_{0}$ and this solution is unique in distribution.

Let $\tau_{r}^{n}=\inf \left\{t:\left|X^{n}(t)\right| \geq r\right\}$. The derivation of the convergence $X^{n} \Rightarrow X$ is based on Theorem 7.4.1 of Ethier and Kurtz (1986), as well as Corollary 7.4.2 and its proof. These show that if the limiting SDE has a unique solution (i.e. the martingale problem is well-posed as it is in our case) and we have $X_{0}^{n} \Rightarrow X_{0}$ with

$$
\begin{array}{ll}
\left\|\left(\mathcal{I}_{b^{n}, X^{n}}-\mathcal{I}_{b, X^{n}}\right) \mathbf{1}\left(t \leq \tau_{r}^{n}\right)\right\|_{\infty} \quad \stackrel{P}{\longrightarrow} 0 \\
\left\|\left(\mathcal{I}_{a^{n}, X^{n}}-\mathcal{I}_{a, X^{n}}\right) \mathbf{1}\left(t \leq \tau_{r}^{n}\right)\right\|_{\infty} \quad \stackrel{P}{\longrightarrow} 0
\end{array}
$$

and

$$
\text { for every } \varepsilon, r>0 \quad \sup _{|x| \leq r, \ell} n \mathbb{P}\left(\left|Y_{\ell}^{n}(x)\right| \geq \varepsilon\right) \longrightarrow 0 \text {, }
$$


then $X^{n} \Rightarrow X$. The theorem there only deals with the case of time-independent coefficients, but adding time as an extra coordinate extends the results to the general case.

Condititon (72) follows from the uniform third absolute moment bounds (69) and Markov's inequality. Thus we only need to show (71) as well as the analogous statement for $a$, for which the proof is identical. We do this by bounding the successive uniform-norm distances between

$$
\mathcal{I}_{b^{n}, X^{n}}, \quad \mathcal{I}_{b^{n}, X^{n, L}}, \quad \mathcal{I}_{b, X^{n, L}}, \quad \mathcal{I}_{b, X^{n}}
$$

where $X_{\ell}^{n, L}=X_{K\lfloor\ell / K\rfloor}^{n}$ with $K=\lceil n T / L\rceil$, and $X^{n, L}(t)=X_{\lfloor n t\rfloor}^{n, L}$. In words, we divide $[0,\lfloor n T\rfloor]$ into $L$ roughly equal intervals and then set $X_{\ell}^{n, L}$ to be constant on each interval and equal to the first value of $X_{\ell}^{n}$ occurring there.

If a function $f$ takes countably many values $f_{i}$, then for any $h$ we have

$$
\left\|\mathcal{I}_{h, f} \mathbf{1}\left(t \leq \tau_{r}^{n}\right)\right\|_{\infty} \leq \sum_{i}\left\|\mathcal{I}_{h, f_{i}} \mathbf{1}\left(t \leq \tau_{r}^{n}\right)\right\|_{\infty}
$$

Since $X^{n, L}$ takes at most $L$ values, we have

$$
\left\|\left(\mathcal{I}_{b^{n}, X^{n, L}}-\mathcal{I}_{b, X^{n, L}}\right) \mathbf{1}\left(t \leq \tau_{r}^{n}\right)\right\|_{\infty}=\left\|\mathcal{I}_{b^{n}-b, X^{n, L}} \mathbf{1}\left(t \leq \tau_{r}^{n}\right)\right\|_{\infty} \leq L \sup _{|x| \leq r}\left\|\mathcal{I}_{b^{n}-b, x}\right\|_{\infty}=L o(1)
$$

by (67) where $o(1)$ is uniform in $L$ and refers to $n \rightarrow \infty$. From (68), the other terms satisfy

$$
\begin{aligned}
\left\|\left(\mathcal{I}_{b^{n}, X^{n, L}}-\mathcal{I}_{b^{n}, X^{n}}\right) \mathbf{1}\left(t \leq \tau_{r}^{n}\right)\right\|_{\infty} & \leq T\left\|\left(b^{n}\left(\cdot, X^{n, L}(\cdot)\right)-b^{n}\left(\cdot, X^{n}(\cdot)\right)\right) \mathbf{1}\left(\cdot \leq \tau_{r}^{n}\right)\right\|_{\infty} \\
& \leq c_{r} T\left\|X^{n}-X^{n, L}\right\|_{\infty}
\end{aligned}
$$

The same holds with $b$ replacing $b^{n}$. It now suffices to show that

$$
\mathbb{E}\left\|X^{n, L}-X^{n}\right\|_{\infty}=\mathbb{E} \sup _{\ell}\left|X_{\ell}^{n, L}-X_{\ell}^{n}\right| \leq f(L)
$$

uniformly in $n$ where $f(L) \rightarrow 0$ as $L \rightarrow \infty$. The left-hand side of 73 is bounded by

$$
\mathbb{E} \sup _{\ell}\left|X_{\ell}^{n}-\frac{1}{n} \sum_{k=\lfloor\ell / K\rfloor K}^{\ell-1} b_{n}\left(X_{\ell}^{n}\right)-X_{\ell}^{n, L}\right|+\mathbb{E} \sup _{\ell}\left|\frac{1}{n} \sum_{k=\lfloor\ell / K\rfloor K}^{\ell} b\left(X_{\ell}\right)\right|
$$

and the second quantity is bounded by $T \sup _{\ell, x}\left|b_{\ell}^{n}(x)\right| / L$. The first quantity can be written as $\mathbb{E} M^{*}$ where

$$
M^{*}=\max _{i=0, \ldots, L-1} M_{i}^{*}, \quad M_{i}^{*}=\max _{\ell=0, \ldots, K-1}\left|M_{i, \ell}\right|, \quad M_{i, \ell}=X_{i K+\ell}-X_{i K}-\frac{1}{n} \sum_{k=0}^{\ell-1} b^{n}\left(X_{i K+k}\right) .
$$

Note that for each $i, M_{i, \ell}$ is a martingale. For any martingale with $M_{0}=0$ we have

$$
\mathbb{E} \max _{k \leq n}\left|M_{k}\right|^{3} \leq c \mathbb{E}\left|\sum_{k \leq n} \mathbb{E}\left[\left(M_{k}-M_{k-1}\right)^{2} \mid \mathcal{F}_{k-1}\right]\right|^{3 / 2} \leq c n^{3 / 2} \max _{k \leq n} \mathbb{E}\left[\left|M_{k}-M_{k-1}\right|^{3} \mid \mathcal{F}_{k-1}\right]
$$


The first step is the Burkholder-Davis-Gundy inequality (see Kallenberg (2002), Theorem 26.12) and the second step follows from Jensen's inequality. Therefore 69 implies

$$
\mathbb{E}\left[\left|M_{i}^{*}\right|^{3} \mid \mathcal{F}_{i L}\right] \leq c(n / L)^{3 / 2} n^{\frac{-3}{2}}=c L^{\frac{-3}{2}}
$$

which gives the desired conclusion

$$
\left(\mathbb{E} M^{*}\right)^{3} \leq \mathbb{E}\left(M^{*}\right)^{3} \leq \mathbb{E} \sum_{i=0}^{L-1}\left(M_{i}^{*}\right)^{3} \leq c L^{\frac{-1}{2}}
$$

Letting first $n \rightarrow \infty$ and then $L \rightarrow \infty$ gives $(73)$ and 71 .

Acknowledgments. This research is supported by the NSERC discovery grant program and the Canada Research Chair program (Virág). Valkó is supported by the NSF Grant DMS-09-05820. We thank Hermann Schulz-Baldes for references, Michael Aizenmann and Rowan Killip for many interesting comments and discussions.

\section{References}

M. Aizenman and S. Molchanov (1993). Localization at large disorder and at extreme energies: an elementary derivation Comm. Math. Phys. 157, 245-278.

P. W. Anderson (1958). Absence of diffusion in certain random lattices. Phys. Rev. 109:1492-1505.

S. Bachmann and W. De Roeck (2010). From the Anderson model on a strip to the DMPK equation and random matrix theory. J. Stat. Phys. 139, no. 4, 541564.

J.V. Bellissard, P. D. Hislop, and G. Stolz (2007). Correlations estimates in the lattice Anderson model. J. Statist. Phys. 129, Issue 4, 649-662.

R. Carmona, A. Klein, and F. Martinelli (1987). Anderson localization for Bernoulli and other singular potentials. Comm. Math. Phys. 108, no. 1

J.-M. Combes, F. Germinet, and A. Klein (2009). Generalized eigenvalue-counting estimates for the Anderson model. J. Statist. Phys., 135, 201-216.

F. Delyon, B.Simon, and B. Souillard (1985). From power pure point to continuous spectrum in disordred systems. Annales de l'I.H.P, section A, 42, no. 3, 283-309.

S. N. Ethier and T. G. Kurtz. Markov processes. John Wiley \& Sons Inc., New York, 1986. 
J. Fröhlich and T. Spencer (1983). Absence of diffusion in the Anderson tight binding model for large disorder or low energy. Comm. Math. Phys., 88, 151-184.

M.E. Gertsenshtein and V. B. Vasilev (1959). Waveguide with random non-homogeneities and Brownian motion on the Lobachevskii plane. Theor. Probability Appl., 4:391398, 1959.

G.M. Graf and A. Vaghi (2007). A Remark on the estimate of a determinant by Minami. Lett. Math. Phys., 79, no. 1, 17-22.

I. Ya. Goldsheid, S. Molchanov, and L. Pastur (1977). A pure point spectrum of the stochastic one dimensional Schrödinger operator. Funct. Anal. Appl., 11.

O. Kallenberg. Foundations of modern probability. Springer-Verlag, New York, 2002.

R. Killip (2008). Gaussian fluctuations for $\beta$ ensembles. Int. Math. Res. Not. 2008.

R. Killip and M. Stoiciu (2009). Eigenvalue Statistics for CMV Matrices: From Poisson to Clock via Random Matrix Ensembles. Duke Mathematical Journal 146, no. 3, 361-399.

A. Kiselev, Y. Last, and B.Simon (1998). Modified Prüfer and EFGP transforms and the spectral analysis of one-dimensional Schrödinger operators Comm. Math. Phys., 194, 1-45.

H. Kunz and B. Souillard (1980). Sur le spectre des operateurs aux différences finies aléatoires. Comm. Math. Phys. 78, no. 2, 201-246.

N. Minami (1996). Local fluctuation of the spectrum of a multidimensional Anderson tight-binding model. Comm. Math. Phys. 177, 709-725.

S. Molchanov (1981). The local structure of the spectrum of the one-dimensional Schrödinger operator. Comm. Math. Phys. 78, 429-446.

P.E. Protter. Stochastic integration and differential equations, Springer-Verlag, 2005.

H. Schulz-Baldes (2004). Perturbation theory for Lyapunov exponents of an Anderson model on a strip, GAFA. 14, 1089-1117.

B. Valkó and B. Virág (2009). Continuum limits of random matrices and the Brownian carousel. Inventiones Math., 177:463-508.

B. Virág and B. Valkó (2010). Large gaps between random eigenvalues. Annals of Probability. 38, no. $3,1263-1279$ 
B. Virág and B. Valkó (2009). Random Schrödinger operators on long boxes, noise explosion and the GOE. arxiv:0912.0097

Eugene Kritchevski. Department of Mathematics, University of Toronto, Toronto ON M5S 2E4, CANADA. eugene.kritchevski@utoronto.ca.

Benedek Valkó. Department of Mathematics, University of Wisconsin Madison, WI 53705, USA. valko@math.wisc.edu.

BÁlint Virág. Departments of Mathematics and Statistics. University of Toronto. Toronto ON M5S 2E4, CANADA. balint@math.toronto.edu. 